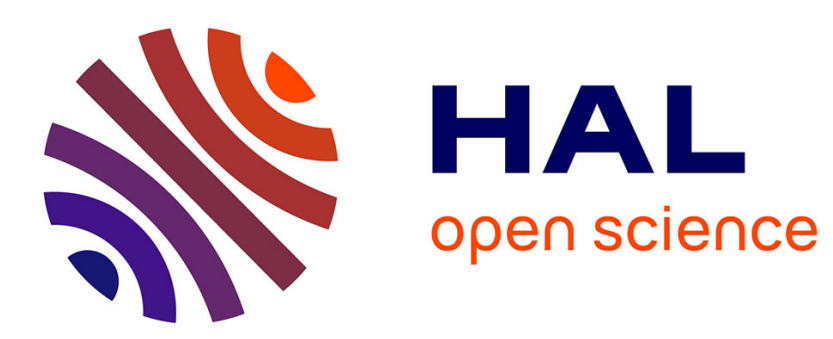

\title{
Robust feedforward-feedback control of a nonlinear and oscillating 2-DOF piezocantilever.
}

Micky Rakotondrabe, Kanty Rabenorosoa, Joël Agnus, Nicolas Chaillet

\section{To cite this version:}

Micky Rakotondrabe, Kanty Rabenorosoa, Joël Agnus, Nicolas Chaillet. Robust feedforward-feedback control of a nonlinear and oscillating 2-DOF piezocantilever.. IEEE Transactions on Automation Science and Engineering, 2011, 8 (3), pp.506-519. 10.1109/TASE.2010.2099218 . hal-00635680

\section{HAL Id: hal-00635680 \\ https://hal.science/hal-00635680}

Submitted on 25 Oct 2011

HAL is a multi-disciplinary open access archive for the deposit and dissemination of scientific research documents, whether they are published or not. The documents may come from teaching and research institutions in France or abroad, or from public or private research centers.
L'archive ouverte pluridisciplinaire HAL, est destinée au dépôt et à la diffusion de documents scientifiques de niveau recherche, publiés ou non, émanant des établissements d'enseignement et de recherche français ou étrangers, des laboratoires publics ou privés. 


\title{
Robust Feedforward-Feedback Control of a Nonlinear and Oscillating 2-DOF Piezocantilever
}

\author{
Micky Rakotondrabe, member, IEEE, Kanty Rabenorosoa, Joël Agnus and Nicolas Chaillet, member, IEEE
}

\begin{abstract}
Many tasks related to the micro/nano-world require, not only high performances like submicrometric accuracy, but also a high dexterity. Such performances are only obtained using micromanipulators and microrobots with multiple degrees of freedom (DOF). Unfortunately, these multi-DOF systems usually present a strong coupling between the different axis making them very hard to control.

The aim of this work is the modeling and control of a 2-DOF piezoelectric cantilever dedicated to microassembly/micromanipulation tasks. In addition to the coupling between the two axis, the piezocantilever is very oscillating and strongly nonlinear (hysteresis and creep). In the proposed approach, the nonlinearity and vibration are first compensated thanks to the feedforward technique. Afterwards, we derive a decoupled model in order to synthesize a linear robust $H_{\infty}$ controller. The experimental results show the efficiency of the proposed approach and their convenience to the micromanipulation/microassembly requirements.
\end{abstract}

Note to Practitioners- The main motivation of this article is the need of both high performances and high dexterity in micromanipulation and microassembly tasks. In such a case, not only a submicrometric accuracy and stability are needed, but also numerous degrees of freedom. For that, in the literature, there exist piezoelectric based structures with 2 or more DOF. Unfortunately, the coupling between its axis, the nonlinearities (hysteresis and creep) and the structure vibration make them very difficult to control and therefore make performances lost. A classical feedback controller can be employed but when the nonlinearities and vibration become strong, it is impossible to synthesize a linear controller. In this paper, we show that the combination of feedforward techniques, to minimize the nonlinearities and vibration, and feedback techniques makes possible to reach the high performances required in micromanipulation/microassembly. We notice that the proposed approach can also be applied to other nonlinear, oscillating and multi-DOF systems, such as piezotubes.

Index Terms-Piezoelectric cantilever, 2 degrees of freedom, coupling, nonlinear, feedforward control, robust control, microassembly/micromanipulation.

FEMTO-ST Institute, UMR CNRS 6174 - UFC / ENSMM / UTBM Automatic Control and Micro-Mechatronic Systems depart., AS2M 24, rue Alain Savary

25000 Besançon - France

Corresponding author: Micky Rakotondrabe,

\{mrakoton $\}$ efemto-st.fr

Paper type: regular paper. First submission: October $5^{t h}, 2009$.

\section{INTRODUCTION}

$\mathbf{T}$ He design and development of microrobots, micromanipulators and microsystems in general used to work in the micro/nano-world (such as for micromanipulation and microassembly) are very different to that of classical systems. At this scale, the systems should have accuracy and resolution that are better than one micron. For instance, fixing a microlens at the tip of an optical fiber with $1 \mu \mathrm{m}$ of relative positioning error or $0.4 \mu \mathrm{rad}$ of orientation error may cause a loss of $50 \%$ of the light flux [1]. In fact, to reach such high performances, the microrobots, micromanipulators and microsystems are developed with smart materials instead of classical motors (example DC motors) and hinges. Smart materials minimizes the mechanical clearances which induce the loss of accuracy. Furthermore, they allow less compact design than hinges because it is possible to design a microsystem with one bulk material. Among the very commonly used smart materials are piezoceramics. Their recognition is due to the high resolution, the short response time and the high force density that they offer.

Beyond the accuracy and resolution, the systems used for microassembly and micromanipulation need to be dexterous. Indeed, when producing hybrid and complex microstructures and MEMS, the used micromanipulators, microrobots and microsystems should be able to perform complex trajectory or should have a complex workspace. For that, they should have higher number of degrees of freedom (DOF). For instance, [2] developped a 2-DOF piezoelectric stick-slip microrobot able to perform angular and linear motion, [3] [4] proposed 3 -DOF microrobots for $x, y, \theta$ motions, [5] proposed a 4 -DOF piezoelectric microgripper and [6] proposes a 6 -DOF dexterous microhandling system. In fact, it has been demonstrated that high DOF-number microassembly/micromanipulation systems offer more possibility for complex, hybrid assembled microstructures and MEMS than one-DOF systems [7]- [10].

Among the commonly used microsystems and micromanipulators, piezoelectric microgrippers (called piezogrippers) are especially adapted for microassembly and micromanipulation because of their ability to perform pick and place tasks with submicrometric resolution, and the possibility to control the manipulation force [11]. Most of existing microgrippers are made of two single-DOF piezocantilevers with only an inplane positioning capability [12]- [15]. To fulfill the dexterity requirement, our previous work [5] proposed a high dexterous two-fingered piezogripper (Fig. 1). With its 4 articular DOF, it is able to orient micro-objects or to pick-transport and place them in and out of plane. In-plane means positioning 
in the $y$-axis while out of plane in the $z$-axis (Fig. 1-a). In particular, when the 4-DOF microgripper is particularly combined with external high range linear or rotary systems, the whole micromanipulation/microassembly system becomes itself dexterous [16]. For instance, such micromanipulation system allowed the manipulation of watch screws or the alignment of beam splitters for microspectrometers [7] [17]. In fact, each finger that composes the 4-DOF microgripper is a 2-DOF piezocantilever that is able to move independently in the two orthogonal directions. Notwithstanding, when applying a reference deflection along $y$-axis (resp. $z$-axis) to the piezocantilever, an undesirable deflection is obtained in the $z$ axis (resp. $y$-axis), making a loss of accuracy. This is due to mechanical imperfection of the structure and particularly to the misalignment of the electrodes. In addition to these, the presence of a manipulated object may also cause a coupling between the two axis. Beyond the coupling, the piezocantilever also presents nonlinearities (hysteresis and creep) and vibrations. While the hysteresis and creep also makes lose the accuracy, vibrations generate undesirable overshoots that may destroy the manipulated micro-objects or the actuators themselves. At the end, despite the high dexterity, general performances are lost due to the coupling, nonlinearities and vibrations. It is obvious that the piezocantilever has to be controlled. This control is at low-level and can only be very useful to ameliorate the efficiency of the whole (teleoperated or automated) micromanipulation/microassembly systems. This is why several projects concerning the development of high performances micromanipulation/microassembly stations include the low-level control and the performances improvement of each actuators these last years [18] [19].

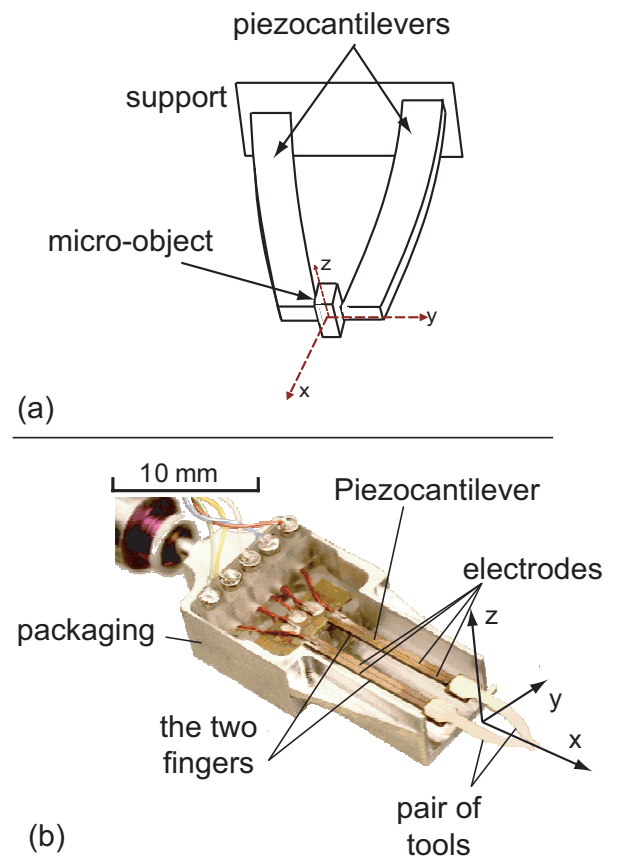

Fig. 1. (a) a piezogripper with 2-DOF piezocantilevers. (b) the microgripper developped in [5].
In the literature, the control of 1-DOF piezocantilevers, including AFM-tubes working on one axis, is at its cruising speed. In open-loop control, both hysteresis and creep were compensated by nonlinear compensators in cascade [20] [21] [22]. Additionaly to the nonlinearity compensation, the vibration was minimized by using dynamic inversion method [23] [24] or inverse multiplicative approach [25]. Openloop control is convenient for sensor-less piezocantilevers, reducing the cost of the whole automated system. However, once external disturbances appear or model uncertainties become large, open-loop control techniques fail and closedloop methods should be used. Different closed-loop control laws were therefore applied and have proved their efficiency in the micro/nano-positioning: integral based control [26], state feedback technique [27], adaptive [28] [29] and robust techniques [30] [31]. The above techniques applied for 1DOF can not be directly extended for 2-DOF piezocantilevers: coupling between axis should be delicately considered. In fact, if the coupling is badly characterized and modeled, the closedloop system may be unstable. In [32], a robust technique was proposed to control a nonlinear 2-DOF nanopositioner. It takes into account the coupling and the nonlinearities. However, when these nonlinearities and the coupling become very large and when the vibration is very badly damped, the technique fails. It is then necessary to propose a new approach that permits to control strongly coupled, hysteretic, creeped and oscillating bi-variable piezocantilevers. Such a technique can be used, not only for multi-DOF piezogrippers dedicated to micromanipulation/microassembly but also for AFM-piezotubes used as a scanner working on two or three axis [33]. The object of this paper is to propose a new technique in that issue. First, we apply a feedforward compensation in order to minimize the effect of the hysteresis and vibration. Afterwards, a model taking into account the coupling and residual nonlinearities is proposed. Finally, a robust feedback control law is synthesized in order to reach the expected performances.

The paper is organized as follows. In section-II, we present the functioning of the 2-DOF piezocantilever that will be controlled. The feedforward technique for compensating hysteresis and vibration is presented in section-III. In sectionIV, we model the obtained system in order to further permit the synthesis of a linear feedback control law. Section-V is dedicated to the synthesis of a robust $H_{\infty}$ controller to reject coupling and uncertainty effects and to reach the specified performances. Finally, conclusions end the paper.

\section{Presentation of the 2-DOF piezocantilever}

\section{A. The piezocantilever working principle}

In this section, we present the 2-DOF piezocantilever that will be controlled. Two of this piezocantilever compose a 4DOF piezogripper (Fig. 1-b) that is able to grip, orient and position micro-objects along $y, z$ and around $x$ axis [5].

The 2-DOF piezocantilever is made up of two piezolayers with 4 local electrodes at its surfaces and one middle electrode for ground (Fig. 2-a). It can be assimilated to a cantilever beam clamped at one end. The two DOF are obtained with a judicious application of voltages on the electrodes. 
Fig. 2-b pictures the functioning of the piezocantilever with cross section views. The structure at rest is presented with solid area and the deformed actuator with dashed lines. $\mathbf{P}$ indicates the polarization of the piezoelectric material. It is reminded that when the electric field (so the applied voltage) is in the same direction than the polarization, the piezolayer contracts. In the first configuration (Fig. 2-c), the four electrodes are set to the same voltage $V_{Z}>0$. The upper layer expands along $x$ while the lower layer contracts, leading to a deflection along $z$. In the second situation (Fig. 2-d), the applied voltages on two adjacent and two opposite electrodes are $V_{Y}$ and $-V_{Y}\left(V_{Y}>\right.$ 0 ). So, while the left part of the piezocantilever expands, the right one contracts leading to a deflection along $y$ axis. Finally, in the last configuration (Fig. 2-e), the electrodes are set at voltages $V_{Z}+V_{Y}$ and $V_{Z}-V_{Y}$, which yields a deflexion in $y$ and $z$ directions. The 2-DOF of the piezocantilever are very interesting for designing piezogrippers with high dexterity. Unfortunately, when a displacement along one axis is desired, a residual displacement along the orthogonal axis appears. This coupling is mainly due to the misalignement of the four electrodes and to the interference between the applied electrical fields. Furthermore, hysteresis and creep nonlinearities as well as vibrations characterize the behavior of the piezocantilever.

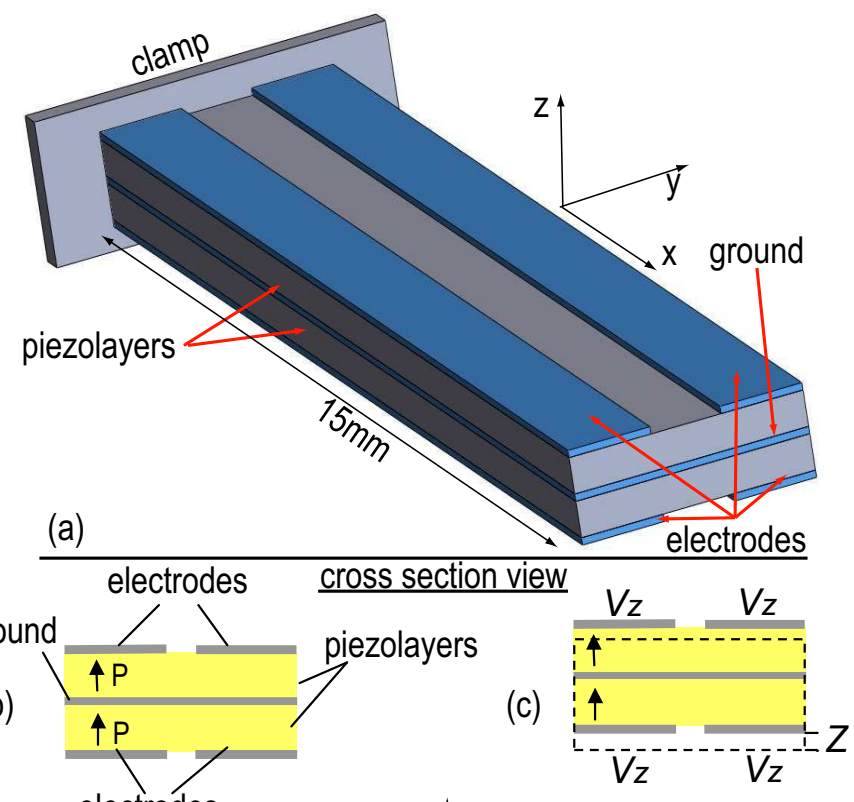

(b) electrodes

(d)

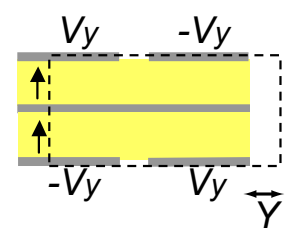

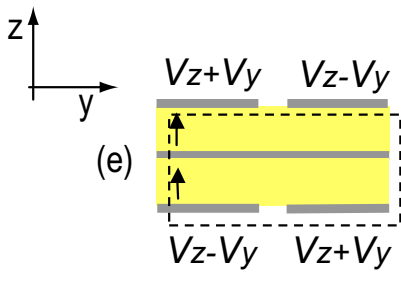

Fig. 2. (a) presentation of the 2-DOF piezocantilever. (b) cross section view of the piezocantilever. (c) achievement of $z$ axis motion. (d) achievement of $y$ axis motion. (e) achievement of both $y$ and $z$ motions.

\section{B. The experimental setup}

Fig. 3 shows the experimental setup. The piezocantilever, made up of PZT-layers, has the following total dimensions: $15 \mathrm{~mm} \times 2 \mathrm{~mm} \times 0.5 \mathrm{~mm}$. Two optical sensors, from Keyence (LK2420), with $10 \mathrm{~nm}$ of resolution and $0.1 \mu \mathrm{m}$ of accuracy are used to measure displacements at the tip of the piezocantilever along $y$ and $z$ axis. We use computer and DSpaceboard materials to acquire measurements and to provide control signals. These real-time materials work with a sampling frequency of $5 \mathrm{kHz}$ which is high enough compared to the bandwidth of the system to be controlled. The control signals that they supply are amplified by a home-made high voltage amplifier having two independent lines. It can supply up to $\pm 200 \mathrm{~V}$ at its output. The Matlab-Simulink ${ }^{\mathrm{TM}}$ software is used to manage the data and signals.

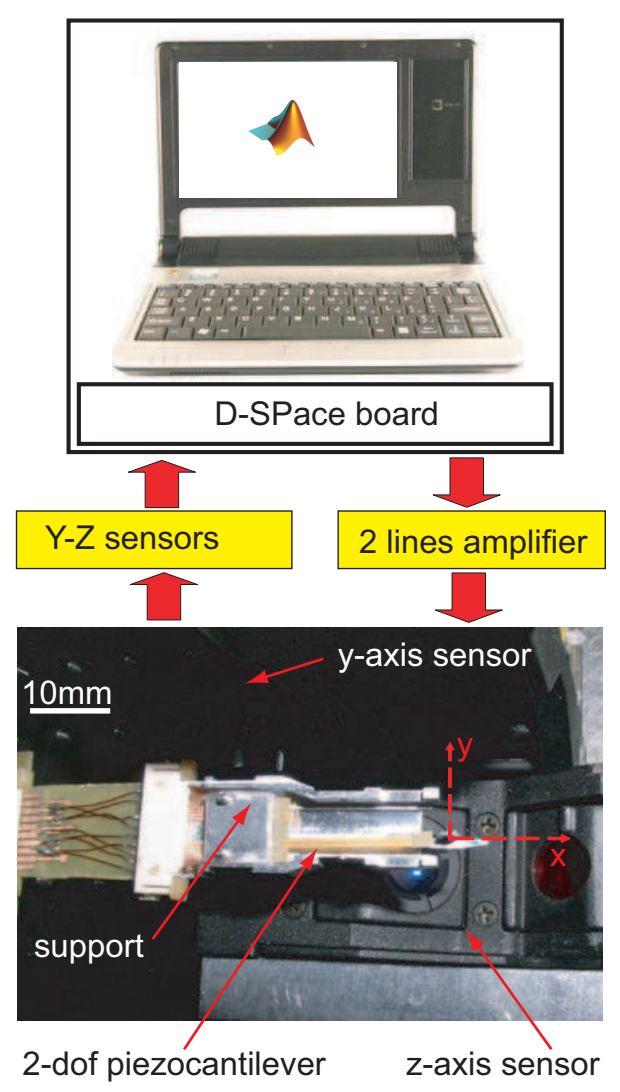

Fig. 3. Experimental setup based on one piezocantilever and two optical sensors.

\section{FEEDFORWARD CONTROL}

In this section, we characterize and compensate the hysteresis and vibration of the piezocantilever. This compensation is necessary in order to linearize the system and to attenuate the vibration and therefore to further make easy the synthesis of a linear controller.

The piezocantilever can be considered as a bi-variable system where inputs are voltages $V_{Y}$ and $V_{Z}$ while outputs are displacements $Y$ and $Z$ (Fig. 4). As advised by previous works [23] [25], the nonlinearities and the vibration analysis can be 
done independently, by choosing properly the frequencies of the input signals. Therefore, we first analyze and compensate the hysteresis. Afterwards, we consider the vibration.

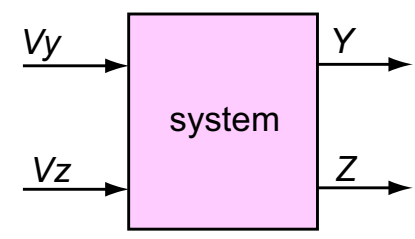

Fig. 4. Block-scheme representing the 2-DOF piezocantilever.

\section{A. Hysteresis compensation}

To characterize the hysteresis, a sine input signal is applied to the system and the resulting output signal is plotted versus the input. The frequency of this input is chosen to be low in order to avoid the effect of the dynamic part on the hysteresis shape. However, it should not be too low in order to avoid the effect of the creep [25] [31]. In our case, we have two input signals $V_{Y}$ and $V_{Z}$. Following our previous characterization work [34], $f_{Y}=f_{Z}=0.05 \mathrm{~Hz}$ is a good choice. Concerning the amplitude, the piezocantilever can be powered by a voltage up to $100 \mathrm{~V}$ but our experiments will be limited to $40 \mathrm{~V}$ (both for $y$ and $z$ axis), which corresponds to the expected range of displacements.

First, we apply a $V_{Y}$ voltage while $V_{Z}$ is set to zero. Two amplitudes $V_{Y}=40 \mathrm{~V}$ and $V_{Y}=20 \mathrm{~V}$ are used. As pictured in Fig. 5-a, a strong hysteresis characterizes the $V_{Y} \rightarrow Y$ transfer. This hysteresis is nearly equal to $17 \%\left(\frac{h}{H} \times 100 \%=\frac{7.5 \mu m}{42 \mu m}\right)$. Furthermore, a residual displacement appears on the $z$ axis (Fig. 5-b). This corresponds to the $V_{Y} \rightarrow Z$ coupling.

Now we set $V_{Y}$ to zero and apply a sine voltage $V_{Z}$. As pictured in Fig. 5-d, a strong hysteresis $(16.67 \%=$ $\left.\frac{20 \mu m}{120 \mu m} \times 100 \%\right)$ also characterizes the $V_{Z} \rightarrow Z$ transfer. Finally, a residual displacement appears on the $y$ axis (Fig. 5c) corresponding to the $V_{Z} \rightarrow Y$ coupling. The asymmetry of the coupling curve is due to the imperfection of the mechanical design of the 2-DOF piezocantilever.

The two hysteresis being too strong, they make difficult the synthesis of further feedback controller. It is then necessary to minimize these hysteresis. The principle used for that is the feedforward compensation based on the inverse model. There are different hysteresis models and compensation techniques for smart materials: the Preisach [35], the Prandtl-Ishlinskii [36] [22] and the Bouc-Wen techniques [37]. We use the Prandtl-Ishlinski model (PI-model) because of the simplicity of its implementation and ease of obtaining a compensator or inverse model.

1) Prandtl-Ishlinskii (PI) hysteresis modeling: in the PI approach, a hysteresis is modeled by the sum of many elementary hysteresis operators, called play operators. Each play operator, denoted by $\gamma_{j}($.$) , is characterized by a threshold r_{j}$ and a
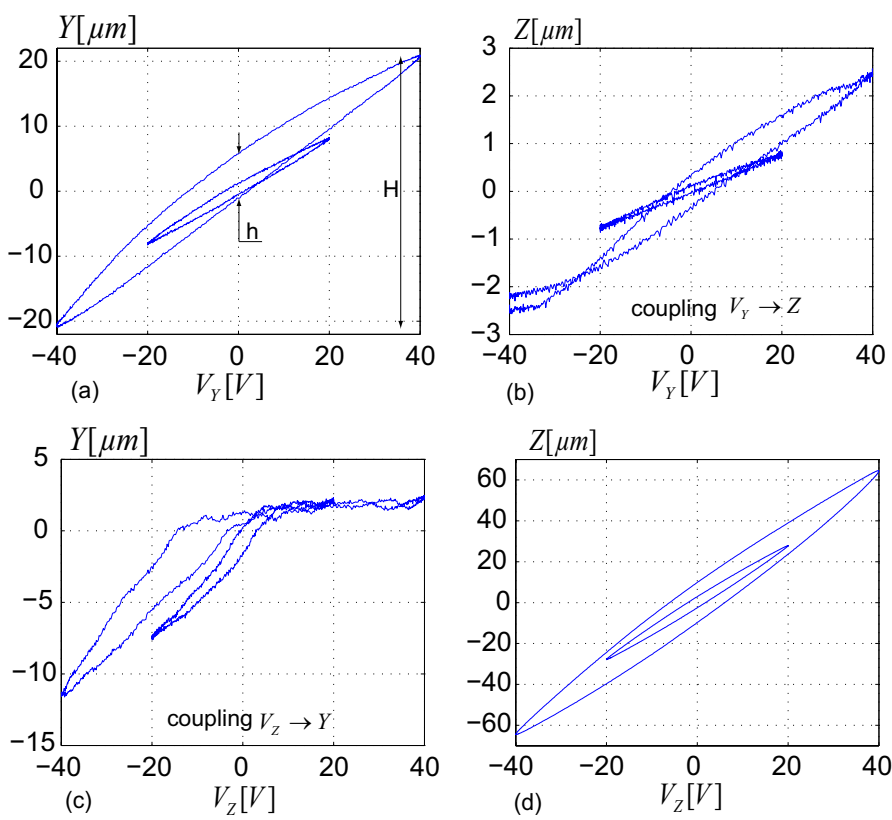

Fig. 5. Hysteresis characteristics of the 2-DOF system piezocantilever.

weighting $w_{j}$ [38]. Thus, the relation between the input $V_{i}$ ( $i \in\{Y, Z\})$ and the output $\delta(\delta \in\{Y, Z\})$ is given by:

$$
\begin{aligned}
& \delta=\sum_{j=1}^{n_{\text {hyst }}} \gamma_{j}\left(V_{i}(t)\right) \\
& =\sum_{j=1}^{n_{\text {hyst }}} \omega_{j} \cdot \max \left\{V_{i}(t)-r_{j}, \min \left\{V_{i}(t)-r_{j}, \delta\left(t^{-}\right)\right\}\right\}
\end{aligned}
$$

where $\delta\left(t^{-}\right)$indicates the value of the output at precedent time and $n_{\text {hyst }}$ the number of play operators. The identification of the parameters $r_{j}$ and $w_{j}$, well described in [25], is done using the maximum voltage input $V_{i}=40 \mathrm{~V}$.

2) Prandtl-Ishlinskii (PI) hysteresis compensation: to compensate a hysteresis that has been modeled with a PI-model, another PI hysteresis model (called hysteresis compensator or PI inverse model) is put in cascade with it. For the 2-DOF piezocantilever, a compensator is put for each axis. In Fig. 6, $Y_{R H}[\mu m]$ is the new input for the $y$ axis while $Z_{R H}[\mu m]$ is for the $z$ axis, and where subscript $R H$ means reference for the hysteresis compensated system.

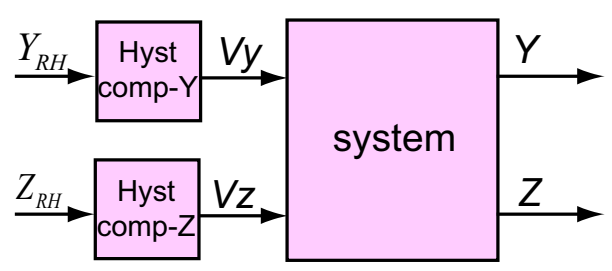

Fig. 6. Block-scheme representing the system with the hysteresis compensators.

Like the PI direct model, each elementary operator of the PI inverse model is characterized by a threshold $r_{k}^{\prime}$ and a 
weighting gain $w_{k}^{\prime}$. They are computed from the parameters $r_{j}$ and $w_{j}$ of the direct model. We have [36]:

$$
r_{k}^{\prime}=\sum_{j=1}^{k} w_{l} \cdot\left(r_{k}-r_{j}\right) \quad ; \quad k=1 \cdots n_{\text {hyst }}
$$

and

$$
\begin{aligned}
& w_{1}^{\prime}=\frac{1}{w_{1}} \\
& w_{k}^{\prime}=\frac{-w_{k}}{\left(w_{1}+\sum_{j=2}^{k} w_{j}\right) \cdot\left(w_{1}+\sum_{j=2}^{k-1} w_{j}\right)} \quad ; \quad k=2 \cdots n_{\text {hyst }}
\end{aligned}
$$

3) Experimental results: first, the PI hysteresis model, described by (Eq. 1), is identified. For a trade-off on accuracy and complexity, we choose $n_{\text {hyst }}=15$. In Fig. 7, the experimental curves and the simulation are plotted. As seen in Fig. 7-b, the identified model for the $V_{Z} \rightarrow Z$ transfer well fits to the real (experimental) hysteresis. However, there is a residual error between the model $V_{Y} \rightarrow Y$ (Fig. 7-a) and the corresponding real hysteresis. This is due to the fact that the real hysteresis is non-symmetrical while the employed model is symmetrical. An adapted model can be used but its compensator has a high complexity for implementation [39]. In addition, the aim is to reduce the hysteresis in order to facilitate the feedback synthesis and the residual error can be considered as uncertainty. This uncertainty will be taken into account during the feedback control design.
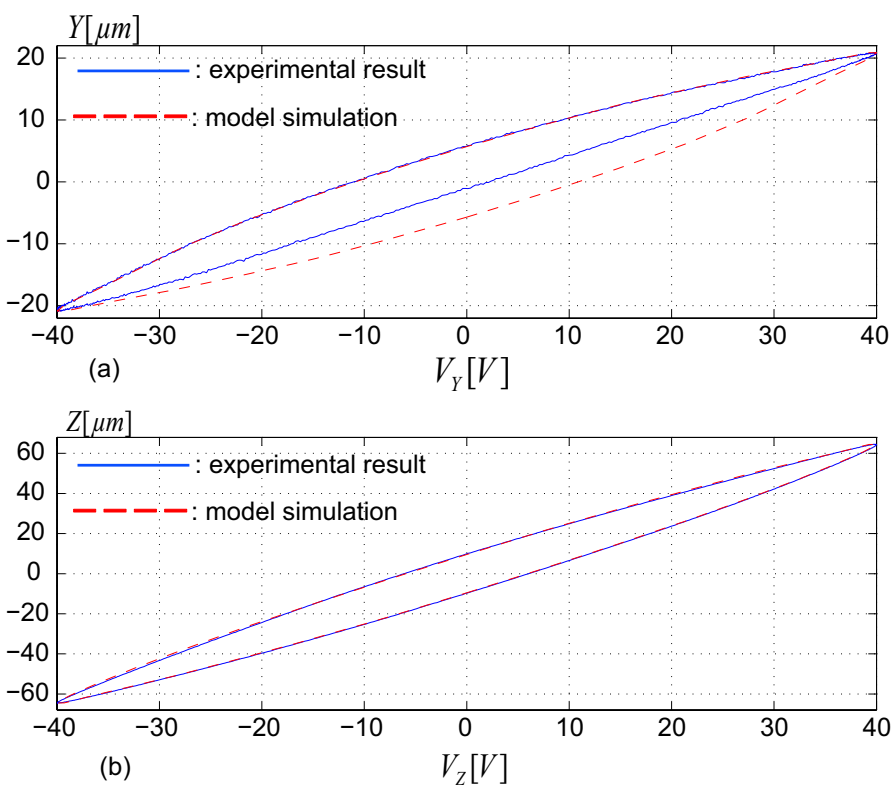

Fig. 7. Experimental results and model simulation of the hysteresis.

The hysteresis compensator has been computed using (Eq. 3), implemented in the Matlab-Simulink ${ }^{\mathrm{TM}}$ software and tested. The experimental process is performed as follows.

First, a sine input reference $Y_{R H}$ with amplitude $20 \mu \mathrm{m}$ is applied while $Z_{R H}$ is set to zero. Fig. 8-a presents the output $Y$. It appears that the hysteresis which was initially
17\% (Fig. ??-a) was reduced into $8.75 \%$. This residual hysteresis is due to the asymmetry of the real hysteresis, already commented above. Fig. 8-b presents the coupling $Y_{R H} \rightarrow Z$. It is shown that this coupling stays unchanged.

After that, a sine reference $Z_{R H}$ with amplitude $60 \mu \mathrm{m}$ is applied while $Y_{R H}$ is set to zero. As pictured in Fig. 8d, the hysteresis which was initially $16.67 \%$ (see Fig. ??-d) is completely removed. However, the coupling $Z_{R H} \rightarrow Y$ becomes slightly larger (compare Fig. ??-c and Fig. 8-c). This coupling will be considered as a disturbance to be removed during the modeling.
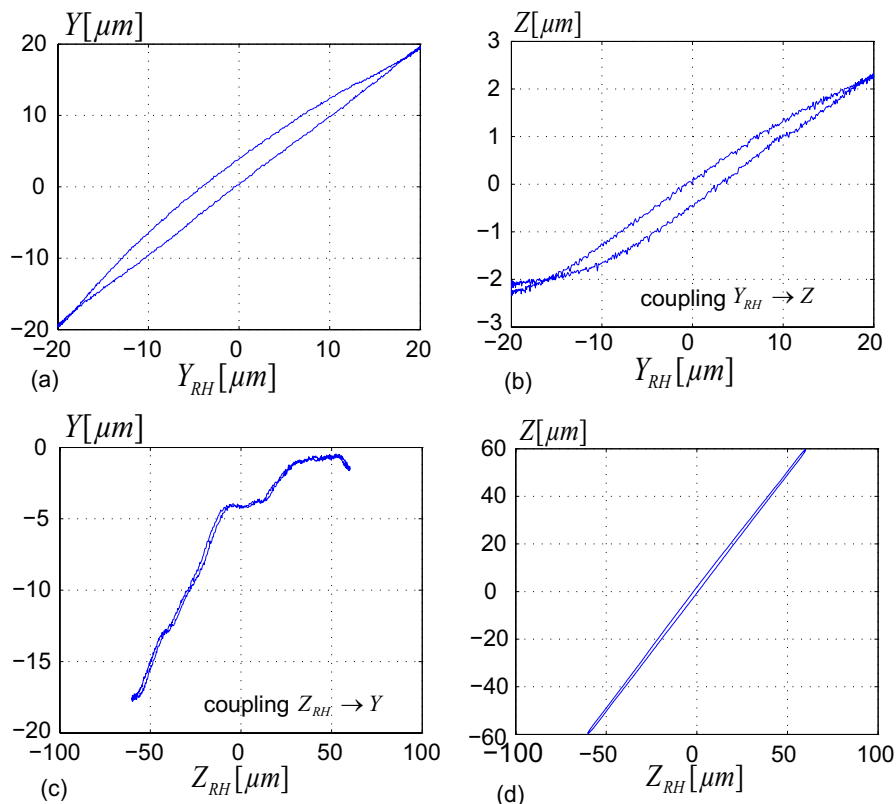

Fig. 8. Experimental results of the 2-DOF piezocantilever with the hysteresis compensator.

\section{B. Vibration compensation}

Now, let us analyze the step responses of the new system represented by the scheme in Fig. 6. For that, we first apply a step $Y_{R H}=20 \mu m, Z_{R H}$ being equal to zero. The response of $Y$ is plotted in Fig. 9-a. Then, we apply a step $Z_{R H}=60 \mu \mathrm{m}$, $Y_{R H}$ being equal to zero. The response of $Z$ is plotted in Fig. 9-d. It appears that the structure is more oscillating and has a badly damped vibration in the $y$ axis than in $z$ axis. The overshoots are $77 \%$ and $4.8 \%$ in $y$ and $z$ axis respectively. The corresponding resonant frequencies are $5400 \frac{\mathrm{rad}}{\mathrm{s}}$ and $2670 \frac{\mathrm{rad}}{\mathrm{s}}$. Finally, the couplings $Y_{R H} \rightarrow Z$ and $Z_{R H} \rightarrow Y$ are pictured in Fig. 9-b and Fig. 9-c respectively.

Similarly to the hysteresis phenomenon, it is hard to synthesis a feedback controller when the vibration characterizing piezocantilevers is too badly damped [34]. This is why we propose to minimize the vibration along $y$ axis. Fig. 10 gives the block-scheme of the 2-DOF piezocantilever with the previous hysteresis compensators and the new vibration 

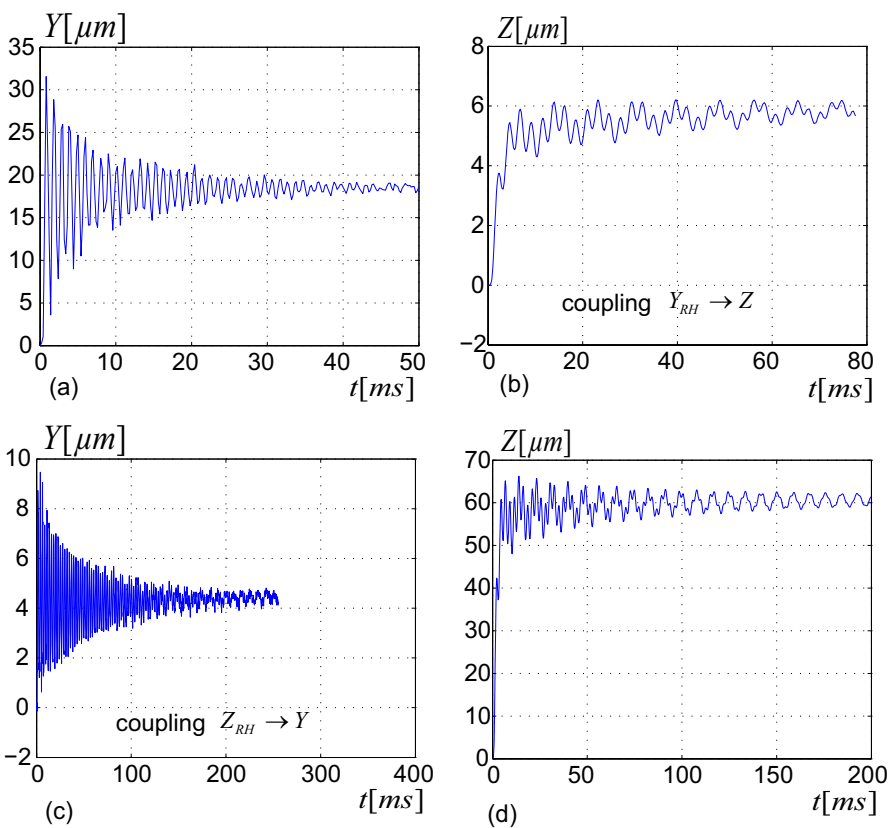

(d)

Fig. 9. Vibration characteristics of the 2-DOF piezocantilever.

compensator. In this, the new references inputs are $Y_{R V}$ and $Z_{R V}=Z_{R H}$. Subsrcipt $R V$ means reference for the vibration compensated system.

To compensate a dynamic part, an inverse dynamic model can be used [23] [24]. This technique necessitates a bistability condition (direct and inverse models stable) on the model. However, such a condition is not always guaranteed for real systems. Another technique to minimize or cancel vibration is input shaping techniques. Input shaping techniques avoid the dynamic inversion and have a simplicity of implementation [25]. There are different kinds of input shaping methods but the one presented here is the Zero Vibration (ZV) method which has the particularity to be simple for computation [40].

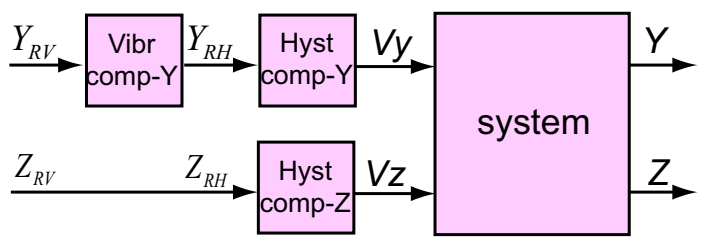

Fig. 10. Block-scheme representing the system with the hysteresis compensators and the vibration compensator.

1) The ZV input shaping technique: When an impulse is applied to an oscillating system, a vibration appears. Let $\omega_{n}$ be the natural frequency and $\xi$ the damping ratio. When a second impulse is applied at time $T_{d}=T / 2$, with $T=\frac{2 \cdot \pi}{\omega_{n} \cdot \sqrt{1-\xi^{2}}}$, the vibration caused by the second impulse can cancel the one caused by the first impulse (Fig. 11-a) if the amplitudes of both are judiciously chosen. For any reference input, the previous sequence of impulses, also called shaper, is convolved with it. Fig. 11-b shows the bloc-diagram of the shaper, which constitutes the vibration compensator and which is convolved with the input reference. For instance, if the reference input is a step, the control signal is a staircase with two steps.

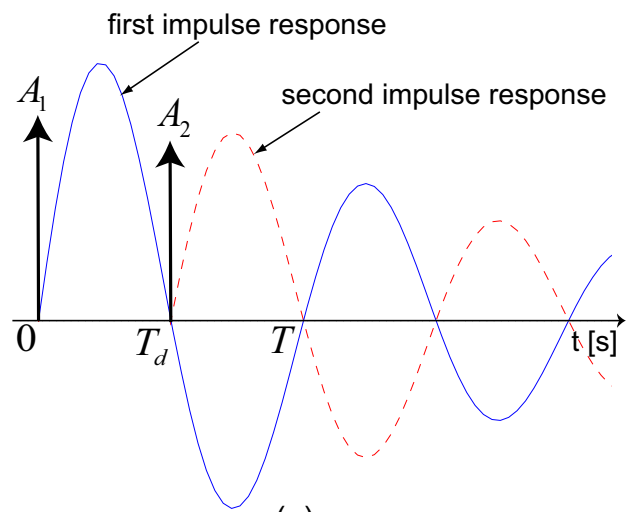

(a)

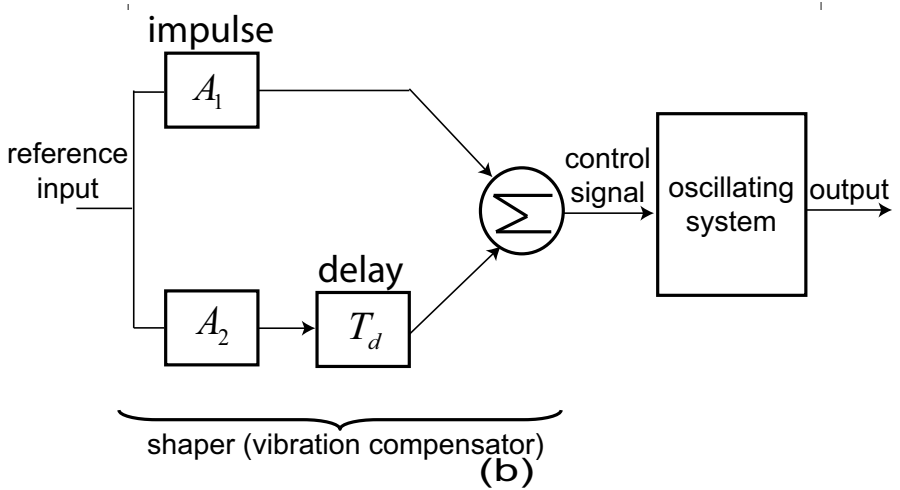

Fig. 11. Principle of the ZV input shaping technique to minimize vibration.

If the identified parameters $\omega_{n}$ or $\xi$ are quite different from the real parameters, a residual vibration will remain after compensation. In fact, the vibration caused by the first impulse will not be exactly cancelled by that of the second one. Therefore, if the overshoots of the two vibrations are very high, the resulting interfered signal may also have a high overshoot. To avoid such a problem, it is advised to use more than two impulses in the shaper. In that case, each impulse amplitude and the corresponding vibration are small. So, the resulting interfered signal will have a lower overshoot if any.

Let an oscillating system be modeled by the following second order model:

$$
\frac{\delta}{\delta_{R}}=\frac{K}{\left(\frac{1}{\omega_{n}}\right)^{2} \cdot s^{2}+\frac{2 \cdot \xi}{\omega_{n}} \cdot s+1}
$$

where $K$ is the static gain.

If $A_{i}$ and $t_{i}$ are the amplitudes of the impulses and their application times (delays), the shaper is computed using the following expressions [40]: 


$$
\begin{gathered}
A_{i}:\left(\begin{array}{c}
A_{1}=\frac{a_{1}}{(1+\beta)^{m-1}} \\
A_{2}=\frac{a_{2}}{(1+\beta)^{m-1}} \\
\vdots \\
A_{m}=\frac{a_{m}}{(1+\beta)^{m-1}}
\end{array}\right) \\
t_{i}:\left(\begin{array}{c}
t_{1}=0 \\
t_{2}=T_{d} \\
\vdots \\
t_{m}=(m-1) \cdot T_{d}
\end{array}\right)
\end{gathered}
$$

where $\beta=e^{-\frac{\xi \cdot \pi}{\sqrt{1-\xi^{2}}}}, m$ is the number of impulses in the shaper, $a_{i}$ indicates the $i^{\text {th }}$ monomial of the polynomial from $(1+\beta)^{m-1}$. We have $a_{1}=1$ and $a_{m}=\beta^{m-1}$.

2) Experimental result: first, we identify the transfer $Y_{R H} \rightarrow Y$. We obtain: $K=0.93, \omega_{n}=6101 \mathrm{rad} / \mathrm{s}$ and $\xi=0.02$. Fig. 12 shows that the identified model reasonably fits to the experimental result.

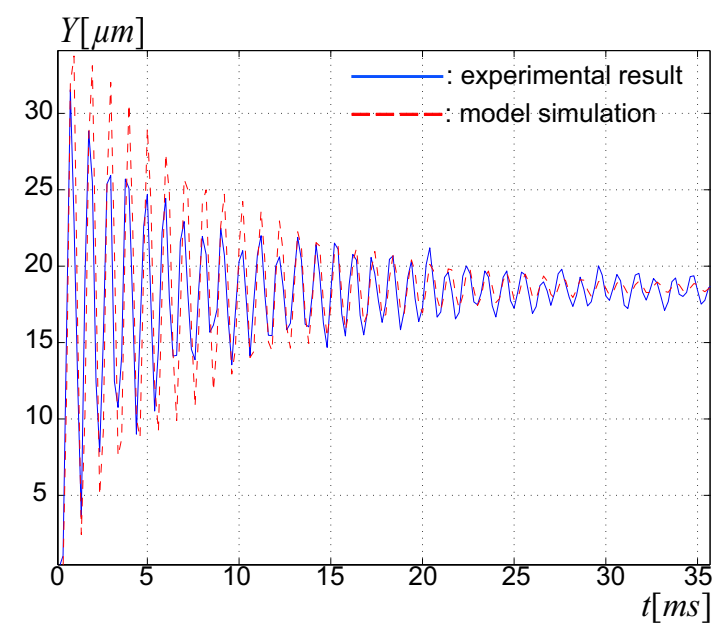

Fig. 12. Step response of the piezocantilever in $Y$-axis: experimental result and model simulation.

Then, different shapers, characterized by different numbers of impulses, were computed and implemented accordingly to Fig. 10. Higher the number of impulses is, lower is the overshoot of the obtained output $Y$, when a step reference input $Y_{R V}$ is applied. However, the complexity of the implemented compensator increases versus the number of impulses. It is therefore unecessary to have a shaper with a high number of impulses. In our case, when the number of impulse is more than 4 , the overshoot stops decreasing drastically. So, we propose to finally choose a shaper with four impulses.

The first experiment concerns the step response on $Y$. In order to compare the results with and without compensator, both results are plotted as in Fig. 13. The figure clearly shows that the overshoot which is $72.22 \%$ without compensator is highly reduced when using the 4 -impulse compensator.

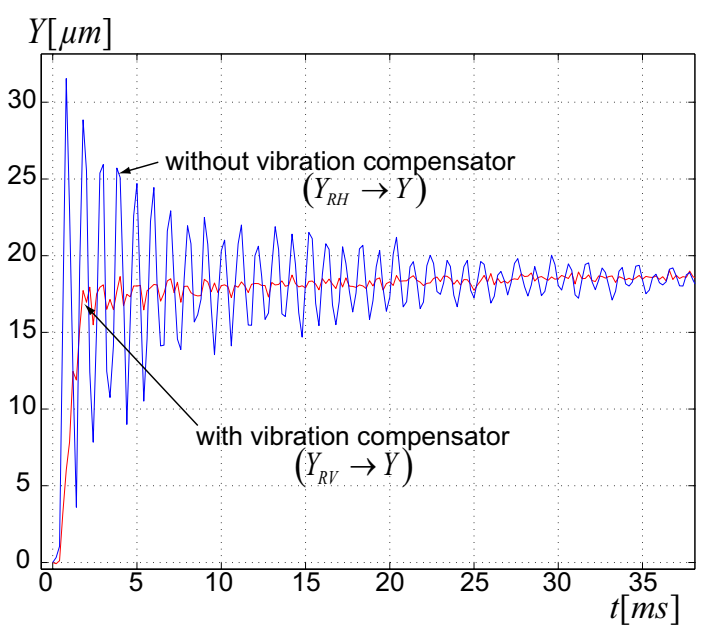

Fig. 13. Experimental step responses of the piezocantilever in $Y$-axis: comparison results of with and without compensator.

After that, a harmonic analysis is performed. For that, a sine input signal is applied and the corresponding output magnitude is plotted. Fig. 14 presents the comparison of the results with and without compensator. It shows that the initial resonance peak is highly damped when we apply the input shaping method.

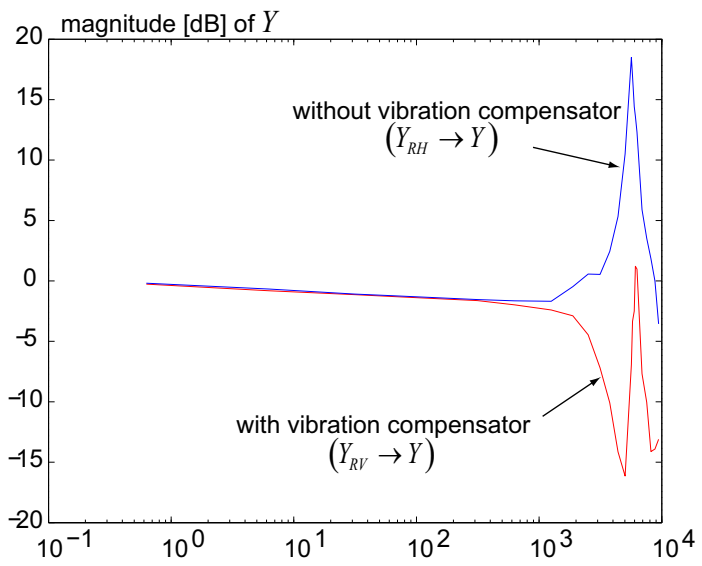

Fig. 14. Experimental harmonic responses of the piezocantilever in $Y$-axis: comparison results of with and without compensator.

\section{Scheme of the new system}

In the previous sub-sections, we have compensated the hysteresis of two axes and the vibration of the $y$ axis using the feedforward techniques. The new system to be modeled and controlled with feedback is now a bi-variable system with smaller hysteresis and vibration, but still with a strong coupling. It has inputs $Y_{R V}$ and $Z_{R V}$ while outputs are $Y$ and $Z$ (Fig. 15). The next section will be dedicated to the modeling of this new system. 


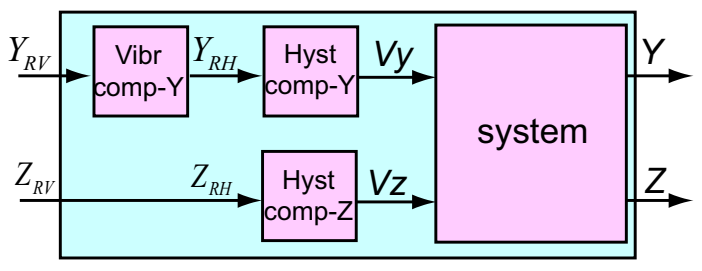

Fig. 15. Block-scheme of the new system to be modeled and controlled with feedback technique.

\section{Modeling}

In this section, we first characterize the system. Afterwards, we propose to model the system by a decoupling technique for $y$ and $z$ axis. The two decoupled models are advantageous relative to one multivariable model because we handle simpler models and therefore synthesize simple controllers. The identification part and the scheme of the nominal model used for the controllers design end the section.

\section{A. Characterization of the system}

The first characterization concerns the step responses. A step input $Y_{R V}=20 \mu m$ is applied while $Z_{R V}$ is set to zero. Fig. 16-a confirms that the vibration on $Y$ is minimized thanks to the previous input shaping technique. The coupling effect $Y_{R V} \rightarrow Z$ gives a final value of $-0.4 \mu m$ (Fig. 16-b). After that, we set $Y_{R V}=0$ and apply $Z_{R V}=60 \mu \mathrm{m}$. While Fig. 16d pictures the resulting $Z$, Fig. 16-c shows that the coupling $Z_{R V} \rightarrow Y$ is strong (final value nearly equal to $4 \mu \mathrm{m}$ ) and very oscillating.
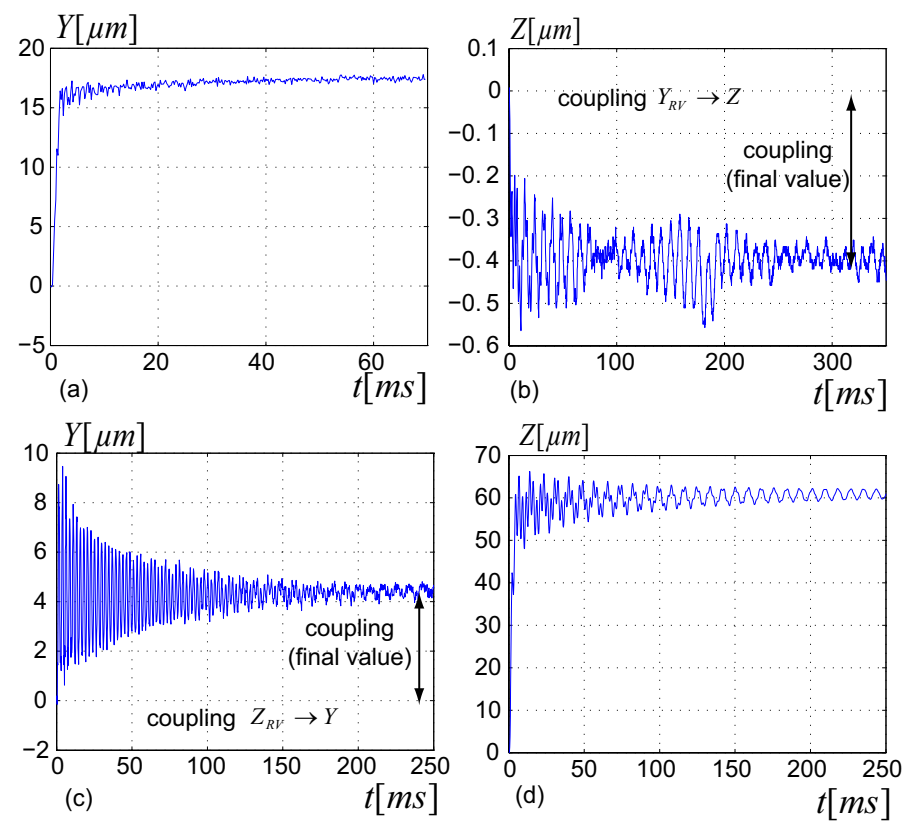

Fig. 16. Step response of the compensated 2-DOF piezocantilever.

The next characterization concerns the static plane behaviors, ie. the input-output plane. For that, a sine input $Y_{R V}$ with amplitude $20 \mu m$ is applied. In parallel, three constant values of $Z_{R V}$ are applied: $0 \mu \mathrm{m}, 30 \mu \mathrm{m}$ and $60 \mu \mathrm{m}$. The response on $Y$, plotted in Fig. 17-a, shows the residual hysteresis in $Y_{R V} \rightarrow Y$. The effect of the constant $Z_{R V}$ on the (three hysteresis) curves is that they are slightly angled and shifted. A sine input $Z_{R V}$ with amplitude $60 \mu m$ is now applied. Three constant values are given to $Y_{R V}: 0 \mu \mathrm{m}, 10 \mu \mathrm{m}$ and $20 \mu \mathrm{m}$. The response of $Z$, plotted in Fig. 17-d shows the linearity of the direct transfer $Z_{R V} \rightarrow Z$. The applied constant $Y_{R V}$ affects the three linear curves by a slight angle.

Fig. 17-b presents the coupling transfer $Y_{R V} \rightarrow Z$. It confirms that the effect of $Y_{R V}$ on $Z$ is negligible. Indeed, we have:

$$
\frac{\partial Z}{\partial Y_{R V}} \simeq 0, \quad \forall Z_{R V}
$$

Fig. 17-c presents the coupling transfer $Z_{R V} \rightarrow Y$, which can be approximated by a linear function such as:

$$
\frac{\partial Y}{\partial Z_{R V}} \simeq \frac{12 \mu m}{60 \mu m}=0.2=a_{Z Y}, \quad \forall Y_{R V}
$$
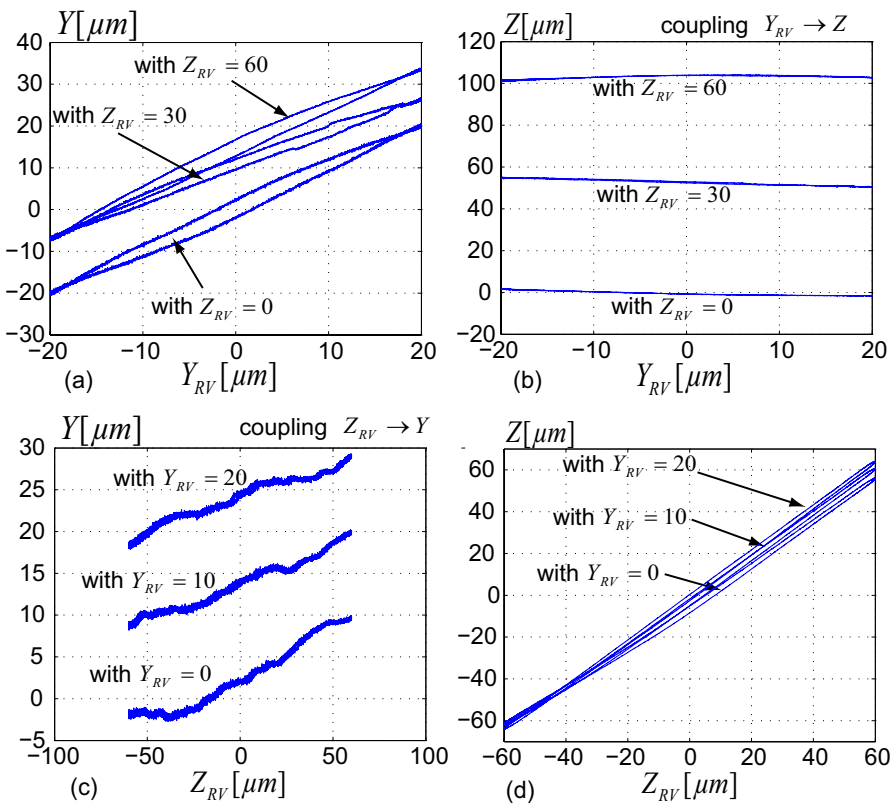

Fig. 17. Characteristics in the static plane of the compensated 2-DOF piezocantilever.

Finally, the last characterization concerns the creep effect. The creep is defined to be the drift appearing after the end of the transient part. These transient parts were presented in Fig. 16. To characterize the creeps, a step input $Y_{R V}=20 \mu m$ (with $Z_{R V}=0$ ) is first applied, afterwards a step input $Z_{R V}=60 \mu m$ (with $Y_{R V}=0$ ) is applied. The outputs are acquired for a long duration: $600 s$, and the curves are given in Fig. 18. The direct transfers $Y_{R V} \rightarrow Y$ and $Z_{R V} \rightarrow Z$ provide creeps of $4 \mu \mathrm{m}$ and $15 \mu \mathrm{m}$ respectively (Fig. 18-a and Fig. 18-d). The coupling transfer $Y_{R V} \rightarrow Z$ provides a creep of $6.5 \mu \mathrm{m}$ (Fig. 18-b). Finally, the creep of the coupling transfer $Z_{R V} \rightarrow Y$ (Fig. 18-c) decreases from $6 \mu \mathrm{m}$ to $5 \mu \mathrm{m}$. 


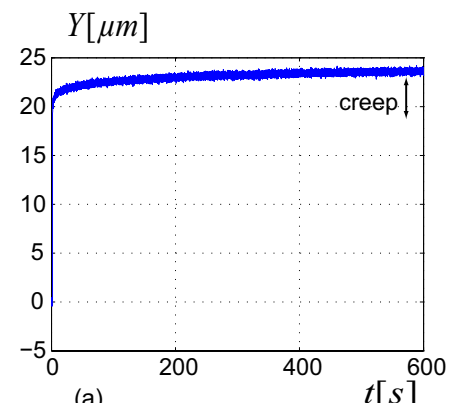

(a)
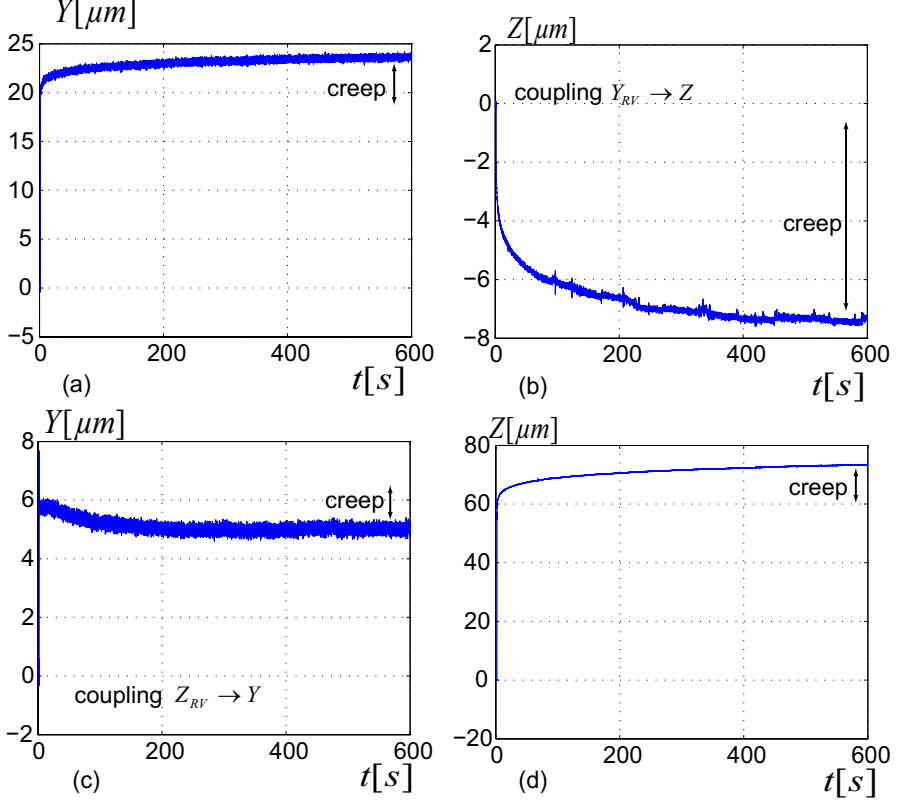

Fig. 18. Creep characteristics of the compensated 2-DOF piezocantilever.

Based on these characterizations, we model the system of Fig. 15 in the next sub-section. The aim is to obtain a simple but convenient model for the synthesis of a feedback controller.

\section{B. The decoupled model}

Let the considered system be represented by the following nonlinear and coupled bi-variable operator:

$$
\left(\begin{array}{c}
Y \\
Z
\end{array}\right)=\Gamma\left(Y_{R V}, Z_{R V}, s\right) \Leftrightarrow\left\{\begin{array}{l}
Y=\Gamma_{Y}\left(Y_{R V}, Z_{R V}, s\right) \\
Z=\Gamma_{Z}\left(Y_{R V}, Z_{R V}, s\right)
\end{array}\right.
$$

where $\Gamma_{Y}$ and $\Gamma_{Z}$ are nonlinear dynamic operators whose inputs are $Y_{R V}$ and $Z_{R V}$. The Laplace variable is denoted by $s$.

1) Equation of the output $Y$ : According to Fig. 17-a and -b and to (Eq. 7), the output $Y$ can be considered as nonlinear relative to $Y_{R V}$ and affine versus to $Z_{R V}$. The dynamic part relating $Z_{R V}$ and $Y$ can be denoted by $D_{Z Y}(s)$ and is characterized by the curve in Fig. 16-c. We deduce:

$$
Y=\Gamma_{Y Y}\left(Y_{R V}, s\right)+a_{Z Y} \cdot D_{Z Y}(s) \cdot Z_{R V}
$$

where the static gain of the coupling $Z_{R V} \rightarrow Y$ is $a_{Z Y}$ and $\Gamma_{Y Y}\left(Y_{R V}, s\right)$ represents a dynamic hysteresis. A dynamic hysteresis is a hysteresis whose shape depends on the rate or frequency of the input. It can be seen from Fig. 17-a that this hysteresis is angled accordingly to the value of $Z_{R V}$, so it is slighlty dependent on the latter. However, because the angle is weak, it will not be considered in the model but in an uncertainty introduced later.

To model the hysteresis, the quadrilateral approximation is applied [31]. It has the advantage to approximate a nonlinear model by a linear one subjected to an uncertainty. As we want to synthesize a simple (linear) controller, this approximation is adapted to our situation. The principle of the quadrilateral approximation is described below.

- The dynamic hysteresis of the piezoactuator can be separated into a static hysteresis and a linear dynamic part. So we have: $\Gamma_{Y Y}\left(Y_{R V}, s\right)=D_{Y Y}(s) \cdot \Gamma_{Y Y}^{s}\left(Y_{R V}\right)$. A static hysteresis is a hysteresis whose shape is independent of the rate or frequency of the input.

- The static hysteresis $\Gamma_{Y Y}^{s}($.$) is approximated by a quadri-$ lateral characterized by a maximal slope $\alpha_{Y \max }$ and a minimal slope $\alpha_{Y \min }$ (Fig. 19).

- Finally, a linear model with direct input multiplicative uncertainty $\Delta \cdot W_{Y e}$ is derived, $W_{Y e}$ being the weighting. For the approximation of $\Gamma_{Y Y}^{s}\left(Y_{R V}\right)$, the nominal static gain is the slope of its axis denoted by $\alpha_{Y}$, and the uncertainty is: $\Delta \in \mathbb{R}$ and $|\Delta| \leq 1$, the weighting $W_{Y e} \in \mathbb{R}$ is chosen to cover the radius $\frac{\alpha_{Y \max }-\alpha_{Y \min }}{2}$.

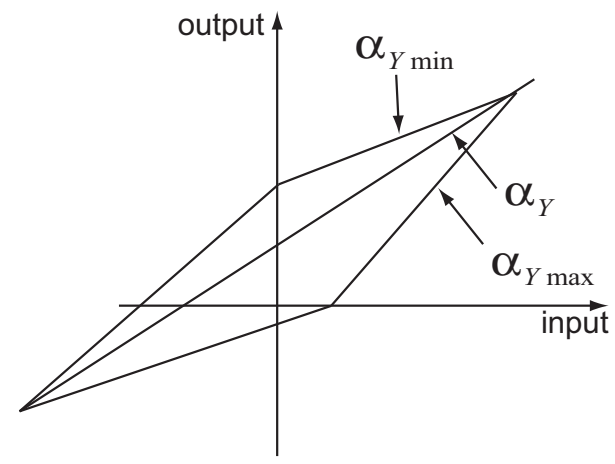

Fig. 19. The quadrilateral approximation of a static hysteresis [31].

The previous model approximates the hysteresis but does not account the creep. It has been demonstrated in [41] that the creep behaves like a fictive external disturbance. So, the equation of $Y$ becomes:

$$
Y=\alpha_{Y} \cdot D_{Y Y}(s) \cdot Y_{R V}+d_{Y}
$$

where:

$$
d_{Y}=a_{Z Y} \cdot D_{Z Y}(s) \cdot Z_{R V}+c_{Y} \cdot F_{Y}+Y_{\text {creep }}
$$

is the external disturbance in the $y$ axis. It is composed of the coupling part $a_{Z Y} \cdot D_{Z Y}(s) \cdot Z_{R V}$, the fictive external disturbance related to the creep part $Y_{\text {creep }}$ and the manipulation force part $c_{Y} \cdot F_{Y}, c_{Y}$ being the compliance. While the direct creep is pictured in Fig. 18-a, the creep due to the coupling denoted by $Y_{c r e e p}^{Z_{R V}}$ is pictured in Fig. 18-c. In the figure, this coupling creep is lower than the final value of $a_{Z Y} \cdot D_{Z Y}(s) \cdot Z_{R V}$, this is why it was not necessary to include it in the disturbance in (Eq. 11).

2) Equation of the output $Z$ : According to Fig. 17-c and -d and to (Eq. 6), the output $Z$ is linear relative to $Z_{R V}$ and the coupling $Y_{R V} \rightarrow Z$ is negligible:

$$
Z=\alpha_{Z} \cdot D_{Z Z}(s) \cdot Z_{R V}
$$


where $\alpha_{Z}$ is the static gain identified from Fig. 17-d and $D_{Z Z}$ is a linear dynamic part identified from the step response given in Fig. 16-d.

Let $Z_{\text {creep }}$ be the direct creep pictured in Fig. 18-d, and $Z_{c r e e p}^{Y_{R V}}$ be the coupling creep due to $Y_{R V}$ in Fig. 18-b. Once again, these creeps can be considered as external disturbances. Finally we have:

$$
Z=\alpha_{Z} \cdot D_{Z Z}(s) \cdot Z_{R V}+d_{Z}
$$

where $d_{Z}$ is the final external disturbance along $z$ axis. It is given by:

$$
d_{Z}=c_{Z} F_{Z}+Z_{\text {creep }}+Z_{\text {creep }}^{Y_{R V}}
$$

with $F_{Z}$ being the manipulation force along $z$ axis and $c_{Z}$ the compliance.

\section{Summary of the modeling}

Fig. 20 sums up the decoupling technique as we have proposed. The initial nonlinear and coupled model in (Eq. 8) has two coupled equations, one for $Y$ and one for $Z$. The linear and decoupled two models were obtained as follows.

- For $Y$, we have brought the coupling (Fig. 17-c), the direct creep (Fig. 18-a) and the manipulation force together in a disturbance. The residual hysteresis (Fig. 17a) was approximated by the quadrilateral approach and has derived an uncertainty of model parameter.

- For $Z$, the direct creep (Fig. 18-d), the coupling creep (Fig. 18-b) and the external force were considered as disturbance. The slight angle due to the coupling (Fig. 17d) was considered as a model uncertainty.

We notice that if another piezocantilever with different characteristics (sizes, etc.) is used, the proposed decoupled and linear model is still valid. One has only to perform first the characterization process as presented in section-IV-A.

\section{Identification}

1) Static gains: the static gain $\alpha_{Y}$ was identified using Fig. 17-a and the principle shown in Fig. 19. The whole three hysteresis curves of Fig. 17-a were used to choose the maximal and minimal slopes. However, to choose the nominal gain, we only use the hysteresis obtained at $Z_{R V}=0$. Concerning the static gain $\alpha_{Z}$, we use Fig. 17-d. Finally, we introduce a weighting $W_{Z e}$ for uncertainty related to the fact that the three curves in Fig. 17-a are slightly angled themselves. We obtain:

$$
\left\{\begin{array}{l}
\alpha_{Y}=1.01 \\
W_{Y e}=0.2 \\
\alpha_{Z}=0.99 \\
W_{Z e}=0.002
\end{array}\right.
$$

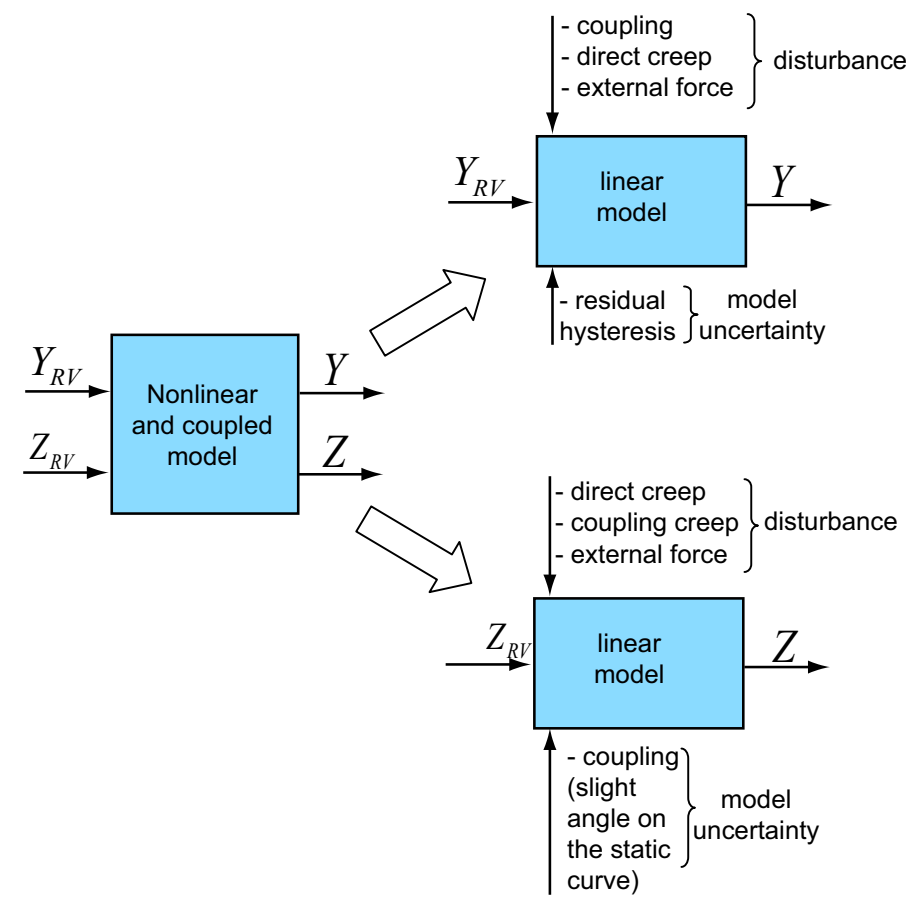

Fig. 20. Summary of the decoupling technique.

2) Dynamic parts: using the step responses in Fig. 16-a and -d, we apply an ARMAX (Auto Regressive Moving Average with eXternal inputs)) model and Matlab software [42] to identify $D_{Y Y}$ and $D_{Z Z}$. Fig. 21 presents the step responses of the identified models compared with the experimental result. We have:

$$
\left\{\begin{array}{l}
D_{Y Y}(s)=\frac{-235\left(s-1.5 \times 10^{4}\right)(s+50.7)}{(s+46.6)\left(s^{2}+3378 s+4.5 \times 10^{6}\right)} \\
D_{Z Z}(s)= \\
\frac{0.002\left(s^{2}+1.43 \times 10^{4} s+6.5 \times 10^{7}\right)\left(s^{2}-5195 s+6.9 \times 10^{7}\right)}{(s+2388)(s+544)\left(s^{2}+49.6 s+7.1 \times 10^{6}\right)}
\end{array}\right.
$$

\section{E. Characterization of the disturbances}

1) Disturbance $d_{Y}$ : the disturbance described by (Eq. 11) is first characterized. The aim is to determine the maximal disturbance that may appear in the $y$ axis.

The coupling part $a_{Z Y} \cdot D_{Z Y}(s) \cdot Z_{R V}$ is computed using the maximal input range $Z_{R V}=60 \mu \mathrm{m}$. In the steady-state mode, this coupling part is $4 \mu m$ (Fig. ??-c). The creep $Y_{\text {creep }}$ obtained at the maximal input $Y_{R V}=20 \mu \mathrm{m}$ is nearly $6 \mu \mathrm{m}$ (Fig. 18-a). The compliance has been identified in a previous work, we have: $c_{Y}=0.625 \mu \mathrm{m} / \mathrm{mN}$ [34]. The maximal manipulation force is $20 \mathrm{mN}$. Then, we obtain: $c_{Y} \cdot F_{Y}=$ $12.5 \mu \mathrm{m}$. Therefore, the maximal disturbance along $y$ is:

$$
d_{Y}=22.5 \mu m
$$

2) Disturbance $d_{Z}$ : here, we characterize the disturbance described by (Eq. 14). 

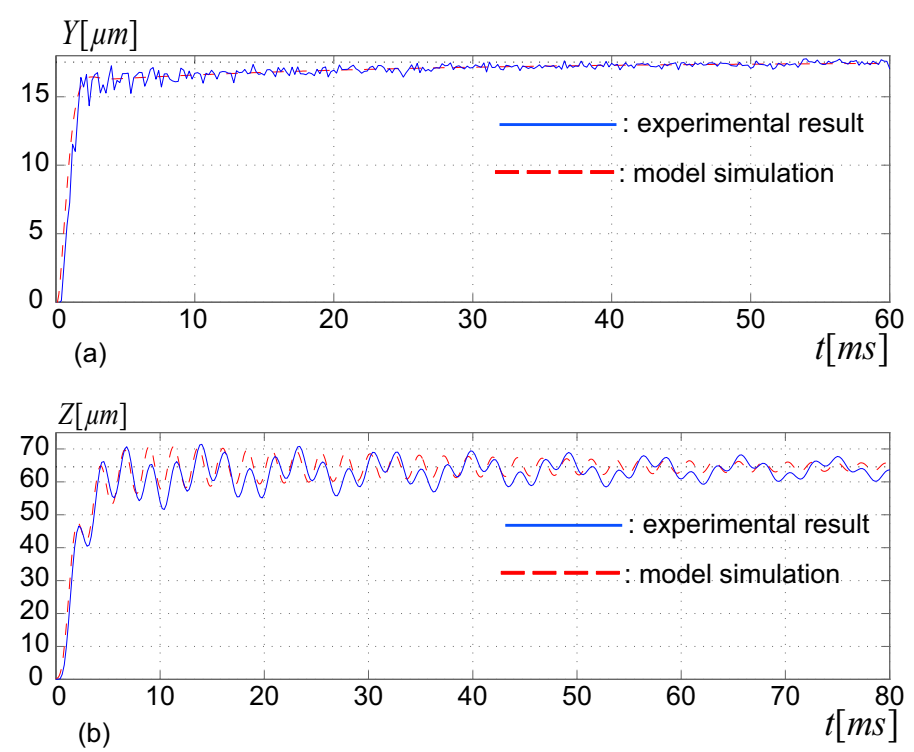

Fig. 21. Experimental results and simulation of the identified transient part.

The identified compliance is $c_{Z}=5 \mu \mathrm{m} / \mathrm{mN}$. With a maximal force of $5 \mathrm{mN}$ along $z$, we have: $c_{Z} F_{Z}=25 \mu \mathrm{m}$. The creep $Z_{\text {creep }}$ is computed using the maximal input range $Z_{R V}=60 \mu m$, we have: $Z_{\text {creep }}=13 \mu m$ (Fig. 18-d). The coupling creep is obtained at $Y_{R V}=20 \mu \mathrm{m}$, we have: $Z_{\text {creep }}^{Y_{R V}}=7.5 \mu m$ (Fig. 18-b). The maximal disturbance along $z$ is then: .

$$
d_{Z}=45.5 \mu m
$$

\section{F. Block-scheme of the model}

The system to be controlled and which is represented by Fig. 15 has been modeled and decoupled in previous subsections. The two decoupled models are given by (Eq. 10) and (Eq. 13). Based on these two models, we can synthesize two separate feedback controllers for $y$ and $z$ axis. The equivalent scheme used for the synthesize is presented in Fig. 22-a. As the two models and their schemes are similar, we use one model and one scheme as presented in Fig. 22-b for the controller synthesis explanations. In the figure, the input control is $\delta_{R V}$ $\left(\delta_{R V} \in\left\{Y_{R V}, Z_{R V}\right\}\right)$, the output is $\delta(\delta \in\{Y, Z\})$, the disturbance is $d\left(d \in\left\{d_{Y}, d_{Z}\right\}\right)$ and the weighting is $W_{e}$ $\left(W_{e} \in\left\{W_{Y e}, W_{Z e}\right\}\right)$. Finally, we have $\alpha \in\left\{\alpha_{Y}, \alpha_{Z}\right\}$ and $D(s) \in\left\{D_{Y Y}(s), D_{Z Z}(s)\right\}$.

\section{ROBUST FEEDBACK CONTROL}

In this section, we synthesize a feedback controller. The objective is to reach the required performances and to reject the disturbance effects that the feedforward techniques cannot account. These disturbances include the creep phenomena, the model uncertainty due to residual hysteresis, the coupling effect and the external applied forces. To account these objectives at the same time, a PID structure would not work because of the limited number of parameters (and therefore the limited
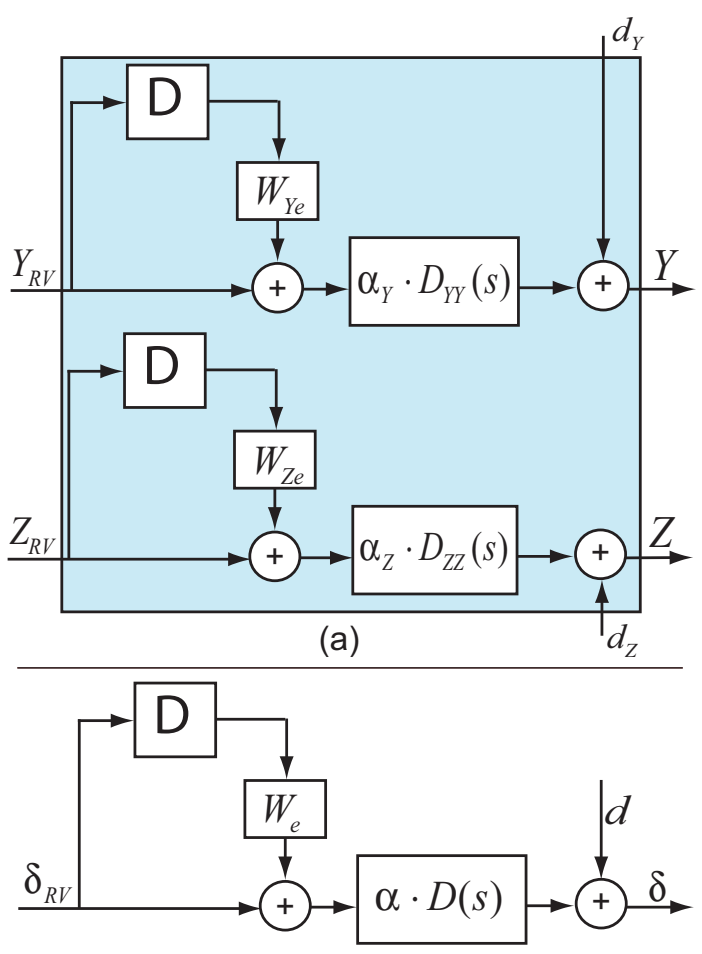

(b)

Fig. 22. Block-scheme of the nominal models used to synthesize the controllers.

number of DOF of tuning). This is why we propose to use a $H_{\infty}$ synthesis technique for the two axis $\left(y\right.$ and $z$ ). A $H_{\infty}$ controller design allows to strongly account the performances specifications and the disturbance rejection. Furthermore, it is possible to take into account the model uncertainty during the synthesis and make the controller robust. The following points will be taken into account during the synthesis:

- the stability has to be ensured despite the presence of uncertainty,

- the effect of the disturbance $d$ should be rejected,

- and the performances required in micromanipulation and microassembly contexts (eg. high accuracy) should be ensured.

\section{A. Scheme and problem formulation}

In $H_{\infty}$ approach, the specifications are represented by weighting functions. Let Fig. 23-a be the closed-loop scheme with the weighting functions used for these specifications. From it, we derive the standard scheme (Fig. 23-b) which presents the interconnection between the augmented system $P(s)$ and the controller $K(s)$. In the figures, $\delta_{R}$ corresponds to the reference input, such as $\delta_{R} \in\left\{Y_{R}, Z_{R}\right\}$. For each axis, a controller $K(s)$ is to be synthesized: $K(s) \in$ $\left\{K_{Y}(s), K_{Z}(s)\right\}$. Finally, $i$ and $o$ are the input and output vector signals respectively. The nominal system is: $G(s)=$ $\alpha \cdot D(s)$.

The standard $H_{\infty}$ problem consists in finding an optimal value $\gamma$ and a controller $K(s)$ stabilizing the closed-loop 


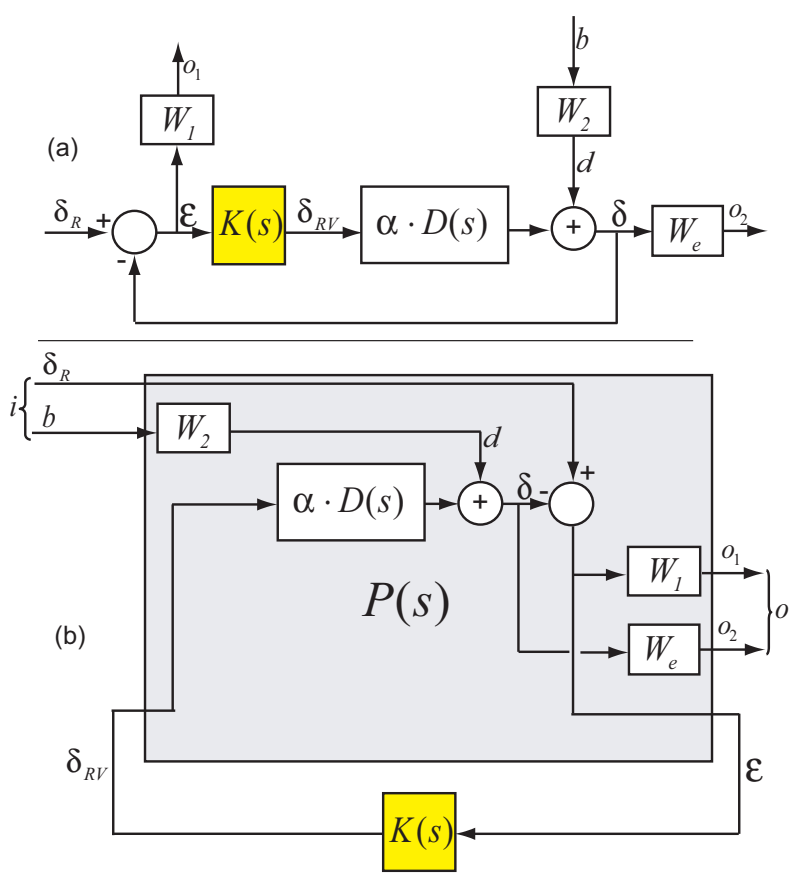

Fig. 23. (a) block-scheme of the closed-loop with the weighting functions. (b) the standard scheme corresponding to the closed-loop.

scheme of Fig. 23-b and guaranteeing the following inequality [43]:

$$
\left\|F_{l}(P(s), K(s))\right\|_{\infty}<\gamma
$$

where $F_{l}(.,$.$) is the lower Linear Fractionar Transformation$ and is defined by $F_{l}(P(s), K(s))=o \cdot i^{-1}$, with $o=$ $\left(\begin{array}{ll}o_{1} & o_{2}\end{array}\right)^{T}$ and $i=\left(\begin{array}{cc}\delta_{R} & b\end{array}\right)^{T}$.

Using Fig. 23-a, we obtain:

$$
\left\{\begin{array}{l}
o_{1}=W_{1} S \delta_{R}-W_{1} S W_{2} b \\
o_{2}=W_{e} K S G \delta_{R}-W_{e} S W_{2} b
\end{array}\right.
$$

where $S=(1+K G)^{-1}$ is the sensitivity function.

Using the condition in (Inequation 19) and the output equations in (Eq. 20), we infer:

$$
\left\{\begin{array}{l}
\left\|W_{1} S\right\|_{\infty}<\gamma \\
\left\|W_{1} S W_{2}\right\|_{\infty}<\gamma \\
\left\|W_{e} K S G\right\|_{\infty}<\gamma \\
\left\|W_{e} S W_{2}\right\|_{\infty}<\gamma
\end{array}\right.
$$

such as the following frequential constraints can be used:

$$
\left\{\begin{array}{l}
|S|<\frac{\gamma}{\left|W_{1}\right|} \\
|S|<\frac{\gamma}{\left|W_{1} W_{2}\right|} \\
|K S G|<\frac{\gamma}{\left|W_{e}\right|} \\
|S|<\frac{\gamma}{\left|W_{e} W_{2}\right|}
\end{array}\right.
$$

To solve the problem in (Inequation 22), we use the GloverDoyle algorithm [44] [45].

\section{B. Weighting functions}

The functions $\frac{1}{W_{1}}$ and $\frac{1}{W_{1} W_{2}}$ are chosen from the specifications on the tracking performances and on the disturbance rejection respectively. Afterwards, the weighting $W_{1}$ and $W_{2}$ are automatically deduced. The functions $\frac{1}{W_{e}}$ and $\frac{1}{W_{e} W_{2}}$ are chosen accordingly to the uncertainty. As $W_{e}$ has already been identified, these two functions are deduced if $W_{2}$ is known from the previous specification.

1) Specifications and weighting for $Y$ : The specifications for tracking performances are as follows.

- the maximal settling time is $40 \mathrm{~ms}$,

- the maximal statical error is $10 \%$,

- the maximal admitted overshoot is $0 \%$.

The specifications for the disturbances rejection are:

- the maximal settling time for rejection is $20 \mathrm{~ms}$,

- the effect of the maximal disturbance $d_{Y}=22.5 \mu \mathrm{m}$ (see (Eq. 17)) should be less than $1 \mu m$, leading to a maximal static error of $4.44 \%$.

From these, we choose:

$$
\left\{\begin{array}{l}
W_{Y 1}=\frac{s+75}{s+0.75} \\
W_{Y 2}=\frac{(s+150)(s+0.75)}{(s+75)(s+6)}
\end{array}\right.
$$

2) Specifications and weighting for $Z$ : The specifications for tracking performances are similar to that of $Y$.

- the maximal settling time is $40 \mathrm{~ms}$,

- the maximal statical error is $10 \%$,

- the maximal admitted overshoot is $0 \%$.

The specifications for the disturbances rejection are:

- the maximal settling time for rejection is $20 \mathrm{~ms}$,

- the effect of the maximal disturbance $d_{Z}=45.5 \mu \mathrm{m}$ (see (Eq. 18)) should be less than $2 \mu m$, leading to a maximal static error of $4.3 \%$.

From these, we choose:

$$
\left\{\begin{array}{l}
W_{Z 1}=\frac{s+75}{s+0.75} \\
W_{Z 2}=\frac{1.25(s+0.1765)(s+0.75)}{(s+75)(s+0.005516)}
\end{array}\right.
$$

\section{Controller computation}

The controllers $K_{Y}(s)$ and $K_{Z}(s)$ for $Y$ and $Z$ respectively were computed. While $K_{Y}(s)$ has an order of $6, K_{Z}(s)$ has an order of 7 . The total order (13) is high and may lead to unwanted numerical error or unstability because of the requirement of high memory and time consumptions in the computer. So, to minimize that, the orders of the controllers have been reduced to 2 and 4 respectively by using the balanced realization technique [46]. We obtain final orders of 2 and 4 :

$$
\left\{\begin{array}{l}
K_{Y}(s)=\frac{0.245(s+531)(s+2.84)}{(s+6)(s+0.75)} \\
\gamma_{Y}=1.43
\end{array}\right.
$$

and 


$$
\left\{\begin{array}{l}
K_{Z}(s)= \\
\frac{539(s+327)(s+0.0059)\left(s 2-8488 s+4.1 \times 10^{6}\right)}{\left(s+1.4 \times 10^{6}\right)\left(s+1.2 \times 10^{4}\right)(s+0.75)(s+0.0055)} \\
\gamma_{Z-o p t}=1.61
\end{array}\right.
$$

\section{Experimental results}

The two controllers were implemented in the MatlabSimulink ${ }^{\mathrm{TM}}$ software, in addition to the feedforward controllers. The complete scheme representing the control of the 2-DOF piezocantilever is shown in Fig. 24. The measurements of $Y$ and $Z$ used for the feedback are provided by the two optical sensors (Keyence) as presented in section-II.

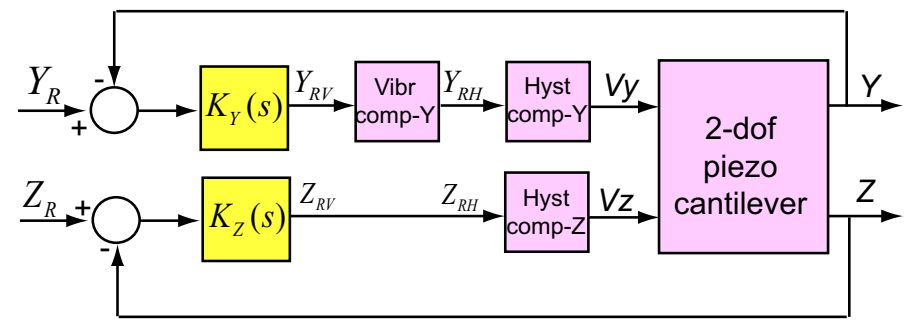

Fig. 24. Block-scheme of the feedforward-feedback control of the 2-DOF piezocantilever.

1) Step responses: first, a series of step references $Y_{R}=$ $\pm 20 \mu \mathrm{m}$ and $Z_{R}= \pm 60 \mu \mathrm{m}$ are applied. The responses are represented in Fig. 25. The coupling $Z_{R} \rightarrow Y$ which is $17 \%$ $\left(=\frac{Y}{Z_{R}}=\frac{10 \mu m}{60 \mu m}=0.17\right)$ is rapidly rejected (Fig. 25-a). The coupling $Y_{R} \rightarrow Z$ is negligible and also rapidly rejected.
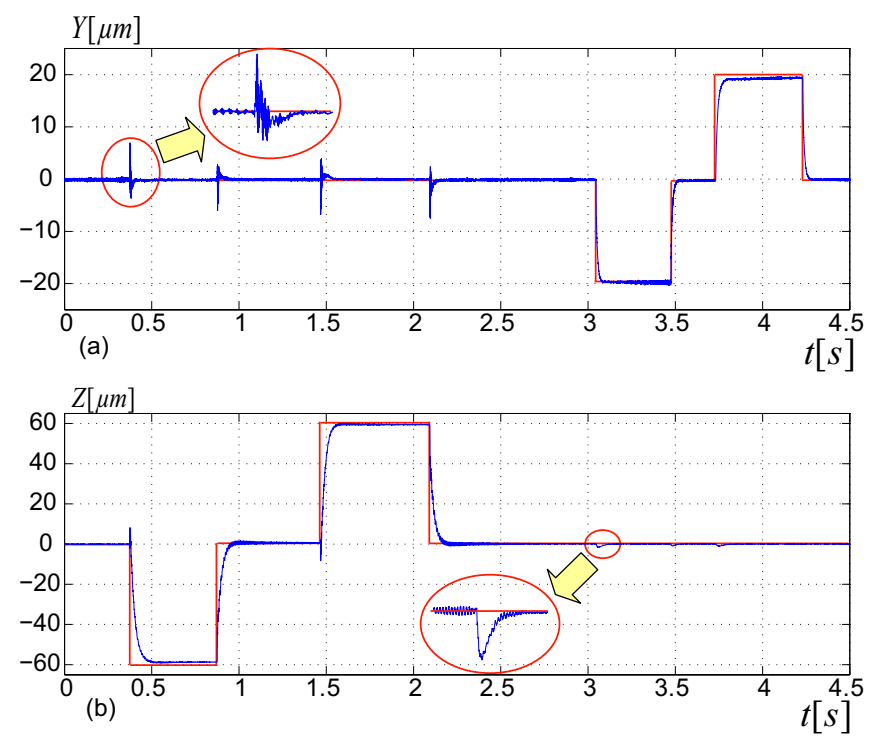

Fig. 25. Steps responses of the controlled 2-DOF piezocantilever.

To analyze the temporal performances, the step responses are zoomed (Fig. 26). It is noticed that the time axis in the latter figures were shifted to start at zero in order facilitate the reading. As seen in the results, the overshoots for the two axis are null, as expected in the specifications. These overshoots were initially $77 \%$ and $4.8 \%$ for $Y$ and $Z$ respectively without the proposed feedback-feedforward controller (see Fig. ??-a and d). Similarly, the static errors tends towards zero, implying a very high accuracy of the controlled piezocantilever. Concerning the response speed, the settling time for $Y$, which was initially $40 \mathrm{~ms}$ (see Fig. ??-a), is unchanged for the controlled piezocantilever. However, the settling time for $Z$, initially more than $100 \mathrm{~ms}$ (see Fig. ??-d), is reduced into $70 \mathrm{~ms}$ when using the proposed control law. This settling time is still higher than the expected in specifications. Such a difference is due to the specifications which were too severe, leading to an optimal $\gamma_{Z}$ more than one. Despite that, the results are well suited for micromanipulation and microassembly tasks.
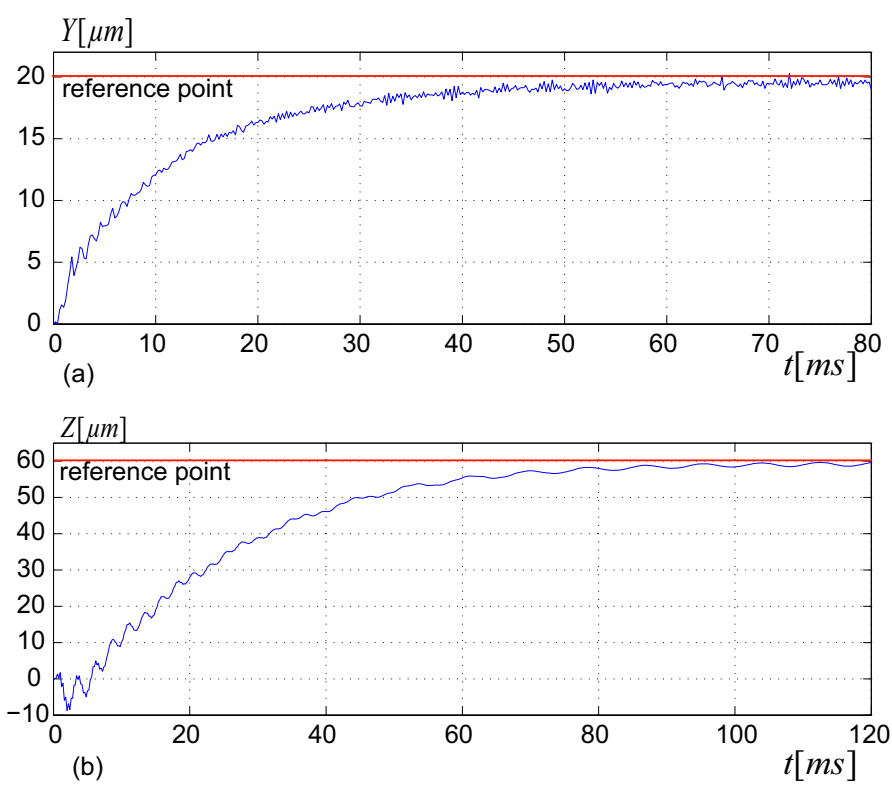

Fig. 26. Zoom of the step responses of the controlled 2-DOF piezocantilever.

2) Harmonic responses: finally, a harmonic experiment is performed in order to evaluate the performances more accurately. For that, a sine wave $Y_{R}$ with $20 \mu m$ of amplitude is applied. Fig. 27-a pictures the transfer $\left(\frac{Y}{Y_{R}}\right)_{d B}$ and $\left(\frac{Z}{Y_{R}}\right)_{d B}$. It shows that whatever the frequency is, the coupling is rejected ( $Z$-magnitude less than $-30 d B$ ). The results also show that no resonance peak higher than $0 d B$ is present for $Y$, indicating the absence of overshoot in the temporal response.

Similarly, a sine input $Z_{R}$ with $60 \mu m$ of amplitude is applied. Fig. 27-b show the responses of $\left(\frac{Y}{Z_{R}}\right)_{d B}$ and $\left(\frac{Z}{Z_{R}}\right)_{d B}$. They show that the effect of $Z_{R}$ on $Y$ is negligible whatever the frequency is, since the coupling is less than $-20 d B$. Finally, once again, the absence of overshoot is demonstrated in the magnitude as there is no resonant peak.

\section{CONCLUSION}

This paper presents the control of a highly coupled, strongly nonlinear (hysteresis and creep) and vibrating 2-DOF piezo- 

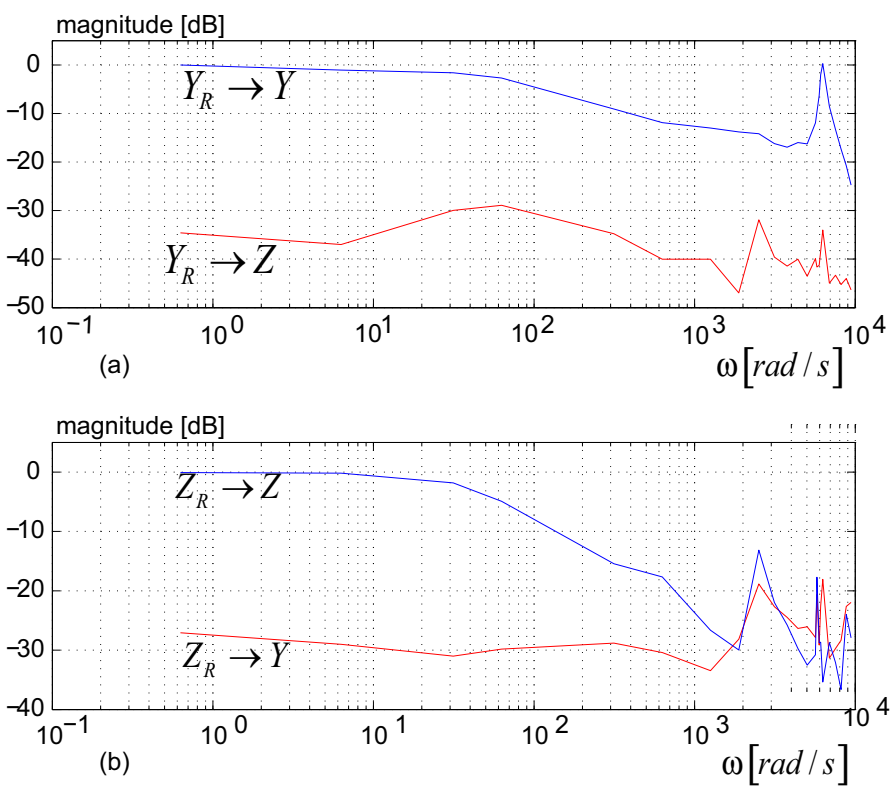

Fig. 27. Harmonic response of the controlled 2-DOF piezocantilever.

cantilever. In order to obtain the required performances, we proposed to combine the feedforward compensation technique and the robust feedback control technique. The feedforward technique was first applied in order to reduce the hysteresis and vibration. Afterwards, a decoupled linear model was developped. The advantage of the proposed model is that it explicitely accounts residual nonlinearities (residual hysteresis, creep) and the coupling by considering them as uncertainty and disturbance to be rejected. Finally, a robust feedback controller was proposed to control the decoupled model. The experimental results show that the proposed approach could provide performances which are suitable for micromanipulation and microassembly aspects. The works presented in this paper will be extended into the control of a microgripper composed of two piezocantilevers.

\section{ACKNOWLEDGMENT}

This work is supported by the French research project NANOROL (ANR $N^{\circ}$ PSIROB07_184846).

\section{REFERENCES}

[1] C. Gartner, V. Blumek, A. Kraplin and T. Possner, "Microassembly processes for beam transformation systems of high-power laser diode bars", MST News I, pp.23-24, 2000.

[2] M. Rakotondrabe, Y. Haddab and P. Lutz, "Development, Modeling, and Control of a Micro-/Nanopositioning 2-DOF Stick-Slip Device", IEEE/ASME Transactions on Mechatronics (T-mech), DOI 10.1109/TMECH.2009.2011134, 2009.

[3] A. Bergander, W. Driesen, T. Varidel, M. Meizoso and J. M. Breguet, "Mobile cm3-microrobots with tools for nanoscale imaging and micromanipulation", Mechatronics Robotics (MechRobo), pp.1041-1047, Aachen Germany, September 2004.

[4] J. Park, D-H. Kim, B. Kim, T. Kim and K. Lee, "Design and performance evaluation of a 3-DOF mobile microrobot for micromanipulation", SpringerLink Journal of Mechanical Science and Technology, pp.12681275,2008

[5] P. de Lit, J. Agnus, C. Clévy and N. Chaillet, "A four-degree-of-freedom microprehensile microrobot on chip", International Journal of Assembly Technology and Management (Assembly Automation), 2004.
[6] Zhou, Q., Korhonen, P., Laitinen, J., Sjövall, S. "Automatic dextrous microhandling based on a 6 DOF microgripper", Journal of Micromechatronics, Vol. 3, No. 3-4, pp. 359-387, 2006.

[7] R. Pérez, J. Agnus, C. Clévy, A. Hubert and N. Chaillet, "Modeling, fabrication and validation of a high performance 2-DOF piezoactuator for micromanipulation", IEEE/ASME Transactions on Mechatronics (Tmech), pp.161-171, April 2005.

[8] N. Dechev and M. Basha, "Robotic microassembly of 3D MEMS structures", IEEE Transactions on Automation Science and Engineering (TASE), Vol.3, pp.207-215, 2008.

[9] C. Clévy, A. Hubert and N. Chaillet, "Flexible micro-assembly system equiped with an automated tool changer", Journal of Micro-Nano Mechatronics, DOI 10.1007.s12213-008-0012-z, 2008.

[10] D. O. Popa, R. Murthy, A. N. Das, "M3- Deterministic, Multiscale, Multirobot Platform for Microsystems Packaging: Design and QuasiStatic Precision Evaluation", IEEE Transactions on Automation Science and Engineering (T-ASE), 2009.

[11] M. Rakotondrabe, C. Clévy and P. Lutz, "Modelling and robust position/force control of a piezoelectric microgripper", IEEE CASE, (International Conference on Automation Science and Engineering), pp.39-44, Scottsdale AZ USA, Sept 2007.

[12] Y. Haddab, N. Chaillet and A. Bourjault, 'A microgripper using smart piezoelectric actuators', IEEE/RSJ IROS, Takamatsu Japan, OctoberNovember 2000.

[13] D. J. Capparelli, M. I. Frecker, T. W. Simpson and A. Snyder, 'Design of a PZT bimorph actuator using a metamodel-based approach', Transactions of the ASME, Journal of Mechanical Design, pp.354-357, 2002.

[14] H. Xinhan, C. Jianhua, W. Min and L. Xiadong ,'A piezoelectric bimorph micro-gripper with micro-force sensing', IEEE ICIA, June-July 2005.

[15] J. Agnus, P. Nectoux and N. Chaillet, 'Overview of microgrippers and design of a micro-manipulation station based on a MMOC microgripper', IEEE CIRA, Espoo Finland, june 2005.

[16] Sylwester Bargiel, Kanty Rabenorosoa, Cédric Clevy, Christophe Gorecki, and Philippe Lutz, "Towards Micro-Assembly of Hybrid MOEMS Components on a Reconfigurable Silicon Free-Space MicroOptical Bench", Journal of Micromechanics and Microengineering, Vol. 10, March 2010, doi:10.1088/0960-1317/20/4/045012.

[17] K. Rabenorosoa, C. Clévy, P. Lutz, A. Das, R. Murthy and D. Popa, "Precise motion control of a piezoelectric microgripper for microspectrometer assembly", ASME IDETC-MNS (International Conference on Micro and Nano Systems), August 2009.

[18] http://www.lab.cnrs.fr/pronomia/

[19] http://nanorol.cnrs.fr/

[20] K. Kuhnen and H. Janocha, "Compensation of the creep and hysteresis effects of piezoelectric actuators with inverse systems", International Conference on New Actuators, pp.309-312, Bremen, June 1998.

[21] H. Jung, J.Y. Shim and D. Gweon, "New open-loop actuating method of piezoelectric actuators for removing hysteresis and creep", Review of Scientific Instruments, 71 (9), pp.3436-3440, 2000.

[22] B. Mokaberi and A. A. G. Requicha, "Compensation of scanner creep and hysteresis for AFM nanomanipulation", IEEE Transactions on Automation Science and Engineering, Vol.5, $N^{o} 2$, pp.197-208, 2008.

[23] D. Croft, G. Shed and S. Devasia, "Creep, hysteresis and vibration compensation for piezoactuators: atomic force microscopy application", ASME Journal of Dynamic Systems, Measurement and Control, 2001.

[24] G. M. Clayton, S. Tien, S. Devasia, A. J. Fleming and S. O. R. Moheimani, "Inverse-feedforward of charge-controlled piezopositioners", Mechatronics, 18, pp.273-281, June 2008.

[25] M. Rakotondrabe, C. Clévy and P. Lutz, "Complete open loop control of hysteretic, creeped and oscillating piezoelectric cantilevers", IEEE Transactions on Automation Science and Engineering (T-ASE), Vol.7(3), pp:440-450, July 2010.

[26] E. Kouno, "PID control", IEEE Control System Magazine, Vol.26, No.1, pp.30-31, January 2006.

[27] Y. Okazaki, "A micro-positioning tool post using a piezoelectric actuator for diamond turning machines", Precision Engineering, Vol.12, pp.151156, July 1990.

[28] C. J. Li, H. S. M. Beigi, S. Li and J. Liang, "Nonlinear piezo-actuator control by learning self tuning regulator", Journal on Dynamics, Systems, Measurements and Control, Vol.115, pp.720-723, December 1993.

[29] X. Tan and J. S. Baras, "Adaptive identification and control of hysteresis in smart materials", IEEE Transactions on Automatic Control (T-AC), Vol.50, No.6, pp.827-839, June 2005.

[30] M. S. Tsai and J. S. Chen, "Robust tracking control of a piezoactuator using a new apprximate hysteresis model", Journal on Dynamics, Systems, Measurements and Control, Vol.125, pp.96-102, March 2003. 
[31] M. Rakotondrabe, Y. Haddab and P. Lutz, "Quadrilateral modeling and robust control of a nonlinear piezoelectric cantilever", IEEE Transactions on Control Systems Technology, Vol.17, Issue 3, pp.528-539, May 2009.

[32] A. Sebastian and S. M. Salapaka, "Design methodologies for robust nano-positioning", IEEE Transactions on Control Systems Technology, Vol.13, No.6, pp.868-876, 2005.

[33] G. Bining, C. F. Quate and Ch. Berger, "Atomic Force Microscope", Physical Review Letters, Vol.56, pp.930-933, 1986.

[34] M. Rakotondrabe, C. Clévy and P. Lutz, "Characterization, modeling and robust control of a nonlinear 2-DOF piezocantilever for micromanipulation/microassembly", IEEE IROS (International Conference on Intelligent Robots and Systems), St Louis MO USA, October 2009.

[35] A. Dubra and J. Massa and C.1 Paterson, "Preisach classical and nonlinear modeling of hysteresis in piezoceramic deformable mirrors", Optics Express, Vol.13, $N^{\circ} 22$, pp.9062-9070, 2005.

[36] K. Kuhnen and H. Janocha, "Inverse feedforward controller for complex hysteretic nonlinearities in smart-materials systems", Control of Intelligent System, Vol.29, $N^{\circ} 3,2001$.

[37] M. Rakotondrabe, "Bouc-Wen modeling and inverse multiplicative structure to compensate hysteresis nonlinearity in piezoelectric actuators", IEEE Transactions on Automation Science and Engineering (T-ASE), DOI.10.1109/TASE.2010.2081979 2010

[38] M. A. Krasnosel'skii and A. V. Pokrovskii, "Systems with hysteresis", Springer-Verlag, Berlin, 1989

[39] K. Kuhnen, "Modeling, identification and compensation of complex hysteretic nonlinearities: a modified Prandtl-Ishlinskii approach", European Journal of Control, Vol.9, No.4, pp.407-418, 2003.

[40] N. C. Singer, W. P. Seering and K. A. Pasch, "Shaping command inputs to minimize unwanted dynamics", Patent $N^{o}$ US-4.916.635, 1990.

[41] M. Rakotondrabe, Y. Haddab and P. Lutz, "Plurilinear modeling and discrete th-synthesis control of a hysteretic and creeped unimorph piezoelectric cantilever", IEEE ICARCV, (International Conference on Automation, Robotics, Control and Vision), pp.57-64, Grand Hyatt Singapour, December 2006.

[42] L. Ljung, "System identfification toolbox", The Matlab user's guide.

[43] G. J. Balas, J. C. Doyle, K. Glover, A. Packard and R. Smith, " $\mu$-analysis and synthesis toolbox", The Mathworks User's guide-3, 2001.

[44] K. Glover and J. C. Doyle, 'State-space formulae for all stabilizing controllers that satisfy an $H_{\infty}$-norm bound and relations to risk sensivity', Systems \& Control Letters, vol.11, pp.167-172, 1988.

[45] J. C. Doyle, K. Glover, P. K. Khargonekar and B. A. Francis, 'Statespace solutions to standard $H_{2}$ and $H_{\infty}$ control problems', IEEE Transactions on Automatic Control, AC 34 Nř8, pp.831-846, 1989.

[46] B. C. Moore, "Principal component analysis in linear systems: controllability, observability and model reduction", IEEE Transactions on Automatic Control (T-AC), AC-26(1), 1981.

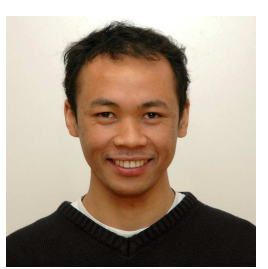

Micky Rakotondrabe (S'05, M'07) received the engineering diploma from the ISTN-ICAM, Lille, France, in 2002, the DEA diploma (M.Sc) in automatic control from the INSA, Lyon, France, in 2003, and the Ph.D. degree in automatic control from the Université de Franche-Comté, Besançon, France, in 2006.

Since 2007, he has been an Associate Professor with the Université de franche-Comté and a researcher with the FEMTO-ST Institute. His field of research concerns the control theory applied to Microsystems and microrobots, including modelling, signal estimation/observation, identification and (feedback and feedofrward) control.

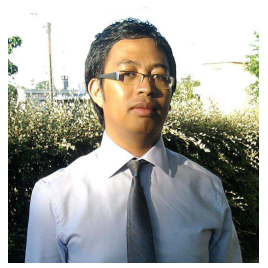

Kanty Rabenorosoa holds a M.Sc. degree (2007) in Electrical Engineering from INSA, Strasbourg, France, and a Ph.D in Microrobotics and Automatic Control (2010) from Université de Franche-Comté, Besançon, France. He is currently an Assistant Professor at the Université de Franche-Comté and at the FEMTO-ST Institute, Besançon France. His research is focused on microrobotic systems and their application to micro-assembly of micro-optical benches. He is also interested by the force control in micro-assembly applications.

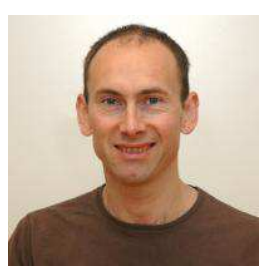

Joël Agnus is engineer in micromechatronic domain since 1996 at the AS2M department of FEMTO-ST. He is involved in several micromanipulation/microassembly projects. His invesigation particularly concerns the design of piezoelectric microgrippers and the integration of force sensors.

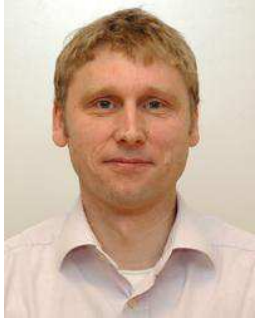

Nicolas Chaillet received his B.S.E. degree in Electrical Engineering from the 'Ecole Nationale Supérieure de Physique', Strasbourg, France, in 1990, and the Ph.D. degree in Robotics and Automation from the University Louis Pasteur, Strasbourg, France, in 1993. In 1995 he became Associate Professor at the University of France-Comté in Besançon, France, working at the 'Laboratoire d'Automatique de Besançon'. Since 2001, he is Professor at the University of Franche-Comté and the head of the Automatic and Micro-Mechatronic Systems Department of the FEMTO-ST Institute since 2008. Since November 2006 he serves as a co-chairman of the IEEE RAS Technical Committee on Micro/Nano Robotics and Automation. His research interests are in Microrobotics and more generally in micromechatronics fields, especially in micromanipulation, microgrippers, smart materials, modeling and control of microactuators and smart microstructures. 